http://dx.doi.org/10.21707/gs.v11.n04a10

\title{
Avanços nos eStudos sobre SEMENTES E PlÂNTUlas de CACTOS Do BRASIL
}

\section{Marcos Vinicius Meiado ${ }^{1 *}$, Ayslan Trindade Lima ${ }^{1}$, Joana Paula Bispo Nascimento ${ }^{1}$, Lidyanne Yuriko SAleme Aona ${ }^{2}$}

\author{
${ }^{1}$ Laboratório de Fisiologia de Sementes, Departamento de Biociências, Universidade Federal de Sergipe. Av. Vereador Olimpio Grande, s/n, Bloco D, \\ Campus Professor Alberto Carvalho, Bairro Porto, Itabaiana, Sergipe, Brasil. CEP: 49510-200. \\ ${ }^{2}$ Herbário do Recôncavo da Babia, Centro de Ciências Agrárias, Ambientais e Biológicas, Universidade Federal do Recôncavo da Babia. Rua Rui Barbosa, \\ 710, Centro, Cruz das Almas, Babia, Brasil. CEP: 44380-000. \\ * Autorpara correspondência: meiado@ufs.br
}

Recebido em 23 de novembro de 2016. Aceito em 04 de abril de 2017. Publicado em 30 de dezembro de 2017.

Resumo - Embora sejam publicados artigos sobre sementes de cactos dos diversos ecossistemas brasileiros há mais de 50 anos, as pesquisas que envolvem o conhecimento acerca da morfologia e fisiologia das sementes e plântulas de Cactaceae são consideradas extremamente recentes no país, pois, durante as quatro primeiras décadas, muito pouco foi publicado sobre essa temática. No Brasil são encontradas 262 espécies de cactos que se distribuem por todo território nacional, porém, até o final da década de 1990, apenas cinco espécies tinham sido estudadas. Dessa forma, o objetivo do presente estudo foi realizar uma revisão bibliográfica para mostrar os avanços nos estudos sobre sementes e plântulas de cactos do Brasil, desde a primeira publicação em 1966 até a presente data. Para atender os objetivos propostos neste estudo, uma extensa revisão bibliográfica foi realizada para a busca de publicações sobre três temas principais: (1) morfologia e anatomia de sementes e plântulas, (2) comportamento germinativo e fatores bióticos e abióticos que afetam a germinação e (3) desenvolvimento inicial e sobrevivência de plântulas de espécies de cactos do Brasil. Nas últimas cinco décadas, 81 artigos sobre esses três temas foram publicados com espécies de cactos que ocorrem no Brasil. Entretanto, a grande maioria desses estudos é recente, sendo que 68\% desses artigos foram publicados de 2010 a 2017. O tema mais estudado foi o efeito dos fatores abióticos na germinação das sementes. Apenas 71 espécies foram estudadas até o momento e alguns padrões já podem ser apontados como, por exemplo, o fotoblastismo positivo da subfamília Cactoideae. Finalmente, incentiva-se a realização de mais estudos sobre essas temáticas, principalmente com representantes da família Cactaceae que ocorrem nos ecossistemas com menor número de espécies estudadas, como o Pampa e o Pantanal.

Palavras-chave: Revis Ão Bibliográfica; Cactaceae; Morfologla; Germinacão; Crescimento Inicial.

\section{AdVANCES IN STUdIES ON SEEDS AND SEEDLINGS OF BRAZILIAN CACTI}

AвstraCt - Although papers about cacti seeds from different Brazilian ecosystems have been published for more than 50 years, research involving the knowledge about the morphology and physiology of Cactaceae seeds and seedlings is considered extremely recent in the country because during the first four decades, few papers have been published about this topic. In Brazil, 262 species of cacti are found that are distributed throughout the national territory, but until the end of the 1990s, only five species had been studied. Thus, the aim of the present study was to carry out a bibliographic review to show the advances in the studies on seeds and seedlings of cacti from Brazil, from the first publication in 1966 to the present date. In order to accomplish the objectives proposed in this study, an extensive bibliographical review was conducted to search for publications on three main topics: (1) morphology and anatomy of seeds and seedlings, (2) germinative behavior and biotic and abiotic factors affecting seed germination and (3) initial development and survival 
of seedlings of Brazilian cacti species. In the last five decades, 81 articles on these three topics have been published with Brazilian cacti species. However, most of these studies are recent, with $68 \%$ of these papers being published from 2010 to 2017. The most studied topic was the effect of abiotic factors on seed germination. Only 71 species were studied and some patterns can already be pointed out, for example, the positive photoblastism of the subfamily Cactoideae. Finally, further studies on these subjects are encouraged, especially with cacti from ecosystems with fewer species studied, such as the Pampa and the Pantanal.

Keywords: Biblographic ReVIeW; CACTACEAE; Morphology; Germination; INITLAL Growth.

\section{AvanCes en los estudios sobre SEMILlas y PLÁNTUlas de CACTUS DE BRASIL}

REsumen - Aunque se publican artículos sobre semillas de cactus de los diversos ecosistemas brasileños desde hace más de 50 años, las investigaciones que involucran el conocimiento acerca de la morfología y fisiología de las semillas y plántulas de Cactaceae son consideradas extremadamente recientes en el país, pues, durante las primeras cuatro décadas, muy poco se ha publicado sobre esta temática. En Brasil se encuentran 262 especies de cactus que se distribuyen por todo el territorio nacional, pero, hasta finales de la década de 1990, sólo cinco especies habían sido estudiadas. De esta forma, se objetivó en el presente estudio realizar una revisión bibliográfica para mostrar los avances en los estudios sobre semillas y plántulas de cactus de Brasil, desde la primera publicación en 1966 hasta la presente fecha. Para cumplir con los objetivos propuestos en este estudio, se realizó una extensa revisión de la literatura para buscar en tres publicaciones principales temas: (1) la morfología y anatomía de las semillas y plántulas, (2) el comportamiento de la germinación y los factores bióticos y abióticos que afectan la germinación y (3) desarrollo inicial y supervivencia de plántulas de especies de cactus de Brasil. En las últimas cinco décadas, 81 artículos sobre estos tres temas fueron publicados con especies de cactus que ocurren en Brasil. Sin embargo, la gran mayoría de estos estudios es reciente, siendo que el 68\% de estos artículos se publicaron de 2010 a 2017. El tema más estudiado fue el efecto de los factores abióticos en la germinación de las semillas. Sólo 71 especies han sido estudiadas hasta el momento y algunos patrones ya se pueden señalar como, por ejemplo, el fotoblastismo positivo de la subfamilia Cactoideae. Finalmente, se incentiva la realización de más estudios sobre esas temáticas, principalmente con representantes de la familia Cactaceae que se encuentran en los ecosistemas con menor número de especies estudiadas, como el Pampa y el Pantanal.

Palabras clave: Retisión Bibliográfica; Cactaceae; Morfología; Germinación; Crecimiento Iniclal.

\section{INTRODUÇÃO}

Embora seja um dos principais símbolos da Caatinga, um ecossistema semiárido localizado na região Nordeste do Brasil, os cactos vêm sendo negligenciados nos estudos sobre a fisiologia de sementes e plântulas, bem como sobre o comportamento germinativo durante boa parte das últimas décadas. Mesmo representando o terceiro maior centro de origem e diversidade da família Cactaceae no mundo (Taylor e Zappi 2004), o Brasil ainda caminha lentamente nos estudos sobre fisiologia de cactos quando comparado a outros países como, por exemplo, os Estados Unidos e o México (Flores et al. 2011). Esse desinteresse pode ser explicado, em partes, pela dificuldade de coleta e identificação das espécies pela maioria dos pesquisadores que estuda a fisiologia de plantas nativas no país. Além disso, ainda pode ser citado o fato de que muitas dessas espécies de cactos estão presentes na Caatinga, um ecossistema exclusivamente brasileiro que é, erroneamente, associado a uma baixa diversidade e que vem sofrendo uma intensa degradação ambiental nos últimos anos (Siqueira-Filho 2012).

De acordo com Zappi et al. (2017), a família Cactaceae está representada no Brasil pela ocorrência de 262 espécies agrupadas em 39 gêneros que se distribuem por todos os domínios fitogeográficos do país. Na Caatinga 
são encontradas 95 espécies que correspondem a 36,3\% dos cactos do Brasil. Cerca de 50\% desses cactos (47 espécies) são endêmicos desse ecossistema (Zappi et al. 2017) e a última espécie descrita dessa família, Melocactus sergipensis N.P. Taylor \& M.V. Meiado, foi descoberta em uma área de Caatinga do Estado de Sergipe, em julho de 2014, e já encontra-se criticamente ameaçada de extinção (Taylor et al. 2014, Meiado et al. 2015).

O primeiro estudo sobre sementes e plântulas de cactos no Brasil foi publicado em meados da década de 1960 e realizado com a espécie Cereus jamacaru DC. ssp. jamacaru, conhecida popularmente como mandacaru (Prisco 1966). Não é de se espantar que essa tenha sido a primeira espécie da família Cactaceae a despertar o interesse dos pesquisadores brasileiros, pois essa planta é amplamente conhecida e possui uma das maiores distribuições geográficas dos cactos que ocorrem no país (Meiado et al. 2012a). Entretanto, até a publicação do artigo "Action of Light on Mandacaru Seed Germination” (Prisco 1966), a informação mais divulgada que se tinha sobre essa espécie vinha da música "O Xote das Meninas", de Luiz Gonzaga, que diz em sua letra: "Mandacaru quando fulora na seca é o sinal que a chuva chega no sertão...". Além de representar uma das estratégias utilizadas pelos profetas da chuva para a previsão da meteorologia popular no sertão (Silva et al. 2013), o mandacaru é uma das espécies mais relacionadas ao conhecimento tradicional do povo sertanejo, que o indica para diversas categorias de uso como, por exemplo, alimento, construção, forragem, místico-religioso, medicinal e ornamental (Lucena et al. 2012, 2013, 2015). Devido a todos os motivos apontados anteriormente, este é o cacto nativo do Brasil mais estudado até o momento, no que se refere à morfofisiologia de sementes e plântulas, ao comportamento germinativo e à produção de mudas de espécies da família Cactaceae no país (Meiado 2012b, Meiado et al. 2012b).

Muitos anos se passaram para que mais um estudo sobre fisiologia de sementes de cactos fosse publicado com a espécie Pereskia aculeata Mill., em meados da década de 1970. Em seu estudo sobre o controle da temperatura na germinação de sementes, Dau \& Laboriau (1974) determinaram a temperatura ótima de germinação de $P$. aculeata $\left(33^{\circ} \mathrm{C}\right)$ e observaram que suas sementes são afotoblásticas quando germinam próximo à temperatura ótima de germinação. Apesar dessa espécie também ser encontrada na Caatinga (Zappi et al., 2017), o estudo de Dau \& Laboriau (1974) foi realizado com sementes coletadas em áreas de Restinga do Estado do Rio de Janeiro, o que pode proporcionar uma diferença no padrão de resposta germinativa ocasionada por variações interpopulacionais. Mais uma década se passou para que outro estudo sobre sementes e plântulas de cactos fosse desenvolvido com a espécie Coleocephalocereus fuminensis (Miq.) Backeb. (Salles 1987), um cacto típico de afloramentos rochosos de áreas de Mata Atlântica nos estados do Espírito Santo, Minas Gerais, Rio de Janeiro e São Paulo (Zappi et al. 2017). Nesse estudo é possível observar a morfologia das sementes e as etapas de desenvolvimento da plântula, desde a germinação até início do desenvolvimento do epicótilo (Salles 1987).

Com uma média extremamente baixa de publicação, sendo observado menos de um artigo publicado a cada década, nos 40 anos posteriores à publicação do primeiro artigo sobre sementes de cactos do Brasil, os estudos sobre fisiologia de sementes e comportamento germinativo de espécies brasileiras ainda podem ser considerados uma ciência recente no país. Dessa forma, o objetivo desta revisão é avaliar os avanços nos estudos sobre sementes e plântulas de cactos que ocorrem no Brasil e reunir todas as informações disponíveis na literatura que possam contribuir para determinar os padrões de respostas fisiológicas de um grupo de plantas extremamente representativo para a flora nacional, devido a sua importância ecológica, econômica e sociocultural.

\section{Material e Métodos}


Para atender os objetivos propostos neste estudo, uma extensa revisão bibliográfica foi realizada para a busca de trabalhos sobre três temas principais: (1) morfologia e anatomia de sementes e plântulas, (2) comportamento germinativo e fatores bióticos e abióticos que afetam a germinação e (3) desenvolvimento inicial e sobrevivência de plântulas de espécies de cactos que ocorrem no Brasil. A revisão bibliográfica compreendeu artigos, livros, relatórios técnicos, monografias, dissertações de mestrado e teses de doutorado publicados nas últimas cinco décadas.

Os estudos relacionados aos três temas investigados nesta revisão foram obtidos por meio de consultas em base de dados nacionais e internacionais, tais como ISI Web of Knowledge, SciELO, Bireme, Periódicos Capes e Google Acadêmico. Todas as buscas foram realizadas utilizando-se as palavras "cacto", "semente", "morfologia", "germinação", "plântula" e "desenvolvimento inicial" (em inglês - "cactus", "seed", "morphology", "germination", "seedling" e "early development” e, em espanhol - "cactus", "semilla", "morfología”, "germinación”, "plántula" e "desarrollo”). Quando um mesmo trabalho abordou dois ou mais temas, este foi incluído em todas as categorias criadas; entretanto, sua citação foi contabilizada apenas uma vez no total de trabalhos consultados.

Estudos realizados com espécies naturalizadas no país como Opuntia ficus-indica Mill., Nopalea cochenillifera (L.) Salm-Dyck e Hylocereus undatus (Haw.) Britton \& Rose, entre outras, também foram incluídos nas análises. As espécies foram classificadas de acordo com a categoria de ameaça e os domínios fitogeográficos de ocorrência, com exceção das espécies naturalizadas. Além disso, os nomes desatualizados de todas as espécies utilizadas como foco de estudo dos trabalhos consultados foram corrigidos com base na Lista de Espécies da Flora do Brasil (Zappi et al. 2017). Todas as referências foram numeradas e organizadas em tabela de acordo com as espécies estudadas para facilitar a localização dos trabalhos consultados.

\section{Resultados e Discussão}

\section{Estudos sobre sementes e plântulas dos cactos do Brasil}

Nas últimas cinco décadas, 81 artigos sobre morfologia e anatomia de sementes e plântulas, comportamento germinativo e fatores bióticos e abióticos que afetam a germinação, além de estudos sobre o desenvolvimento inicial e a sobrevivência de plântulas foram publicados com espécies de cactos que ocorrem no Brasil. Entretanto, a grande maioria desses estudos é recente, sendo que 68\% dos artigos foram publicados de 2010 a 2017. Além disso, espécies da família Cactaceae foram objeto de estudo de nove monografias, 13 dissertações de mestrado e quatro teses de doutorado que investigaram os temas mencionados anteriormente. Alguns desses trabalhos ainda não foram publicados na forma de artigos científicos, o que pode dificultar a divulgação dos resultados presentes nesses estudos realizados com as espécies Cereus fernambucensis Lem. ssp. fernambucensis (Santos 2017), Cipocereus minensis (Werderm.) Ritter ssp. leiocarpus N.P.Taylor \& Zappi (Lopes 2012), Melocactus paucispinus Heimen \& R.J.Paul (Abreu 2008, Resende 2010), Melocactus sergipensis N.P. Taylor \& M.V. Meiado (Santos 2017), Melocactus violaceus Pfeiff. ssp. margaritaceus N.P.Taylor (Bravo Filho 2014, Santos 2017), Melocactus zehntneri (Britton \& Rose) Luetzelb. (Bravo Filho 2014, Santos 2017) e Melocactus x albicephalus Buining \& Brederoo (Cruz, 2011), 
Pilosocereus catingicola (Gürke) Byles \& Rowley ssp. salvadorensis (Werderm.) Zappi (Santos 2017), Pilosocereus gounellei (F.A.C.Weber) Byles \& Rowley ssp. gounellei (Santos 2017), além das espécies do gênero Micranthocereus Backeb. (Aona 2003).

Apenas 71 espécies foram estudadas até o momento, o que corresponde a 27,1\% das espécies de cactos que ocorrem no país (Tabela 1). Dentre todos os cactos avaliados (85 táxons), 47,9\% dos táxons são encontrados na Caatinga, o ecossistema que abriga a maioria das espécies estudada até o momento, seguido pela Mata Atlântica (31,5\%), Cerrado (16,4\%), Pantanal (2,7\%) e Pampa (1,4\%), não sendo encontrado nenhum estudo com espécies de cactos da Amazônia. Vale ressaltar que, a maioria das espécies dos gêneros Arthrocereus A.Berger, Cipocereus F.Ritter e Micranthocereus registrada para os domínios fitogeográficos do Cerrado e da Caatinga (Tabela 1), na realidade, foi coletada em áreas de Campo Rupestre, um tipo de vegetação encontrada no topo de serras e chapadas de altitudes superiores a 900 metros, com afloramentos rochosos onde predominam ervas, gramíneas e arbustos, podendo ser encontradas também algumas arvoretas pouco desenvolvidas.

Cactoideae foi a subfamília mais estudada, sendo encontrados trabalhos sobre 61 espécies agrupadas em 19 gêneros (Tabela 1). Melocactus Link \& Otto é o gênero com o maior número de espécies estudadas até o momento, sendo possível encontrar estudos de cactos desse gênero que ocorrem na Caatinga (10 espécies), no Cerrado (1 espécie) e na Mata Atlântica (1 espécie e 2 subespécies, Tabela 1).

Já a subfamília Opuntioideae foi representada nos estudos consultados por sete espécies agrupadas em quatro gêneros e todas as espécies foram coletadas em áreas de Caatinga, com exceção de Brasiliopuntia brasiliensis (Willd.) A.Berger, uma espécie arbórea coletada em áreas de Mata Atlântica (Meiado et al. 2016). Por sua vez, a subfamília Pereskioideae foi representada apenas pelo gênero Pereskia Mill., sendo duas espécies coletadas na Caatinga, uma na Mata Atlântica e uma no Pantanal.

Em relação às categorias de ameaça de extinção, mais da metade dos táxons estudados até o momento não foram avaliados quanto ao grau de ameaça (NE: 55,9\% e DD: 3,6\%) e mais de $25 \%$ dos táxons encontram-se ameaçados de extinção nas diversas categorias (EN: 13,1\%, VU: 3,6\%, CR: 7,2\%, NT: 2,3\%). Apenas 14,3\% dos cactos estudados até o momento encontram-se na categoria de ameaça de extinção pouco preocupante (LC) (Tabela 1).

\section{Morfologia e anatomia de sementes e plântulas}

Dentre todos os textos consultados, a morfologia de sementes e plântulas de cactos é o segundo mais estudado até o momento. Todos esses trabalhos descreveram a morfologia de sementes e plântulas de 51 espécies distribuídas entre as três subfamílias de Cactaceae (Tabela 1). De acordo com Meiado (2012a), é possível observar uma grande variação morfológica entre as sementes de cactos que ocorrem no Brasil. Sementes de representantes da subfamília Cactoideae são menores que as sementes das espécies de cactos das subfamílias Opuntioideae e Pereskioideae e, a grande maioria das espécies dessa subfamília produz sementes de coloração preta, marrom-escura, ou acastanhada, podendo ser opacas ou brilhantes (Sales et al. 1987, Abud et al. 2010, 2012, 2013, Meiado 2012, Meiado et al. 2012b, 2016). Já as maiores sementes dos cactos que ocorrem no Brasil são produzidas por representantes da subfamília Pereskioideae (Pereskia grandifolia Haw. ssp. grandifolia, com 7,0 x 5,0 mm) e Opuntioideae [B. brasiliensis, com 6,0 x 4,8 mm].

Em seu trabalho sobre a caracterização e a delimitação do gênero Micranthocereus baseadas em caracteres 
morfológicos e moleculares, Aona (2003) incluiu caracteres morfoanatômicos de sementes como, por exemplo, formato e coloração das sementes, presença de polpa funicular, formato das células da testa, presença de crateras nas junções entre as células da testa e de esculturas na superfície celular para propor uma chave de identificação das espécies deste gênero. De acordo a autora, Micranthocereus dolichospermaticus (Buining \& Brederoo) F.Ritter e Micranthocereus estevesii (Buining \& Brederoo) F.Ritter são as únicas espécies que não possuem polpa funicular e, quando essa estrutura está presente em outras espécies do gênero, apresenta a coloração branca. A maioria das espécies desse gênero produz sementes com formato suborbicular e a superfície da testa pode ser lisa, como observado em sementes de $M$. dolichospermaticus, que apresenta as paredes periclinais das células da testa fracamente convexas e sem crateras; ou finamente estriada, como observado em sementes de Micranthocereus purpureus (Gürke) F.Ritter, que também possui as células da testa fracamente convexas, evidenciando a presença de crateras entre as células (Figura 1).

Figura 1. (A) Semente de Micranthocereus dolichospermaticus (Buining \& Brederoo) F.Ritter com formato alongado, paredes periclinais das células da testa fracamente convexas, superfície lisa e sem crateras. (B) Semente de Micranthocereus purpureus

(Gürke) F.Ritter com formato suborbicular, paredes periclinais das células da testa fracamente convexas, superfície finamente estriada com crateras entre as células.

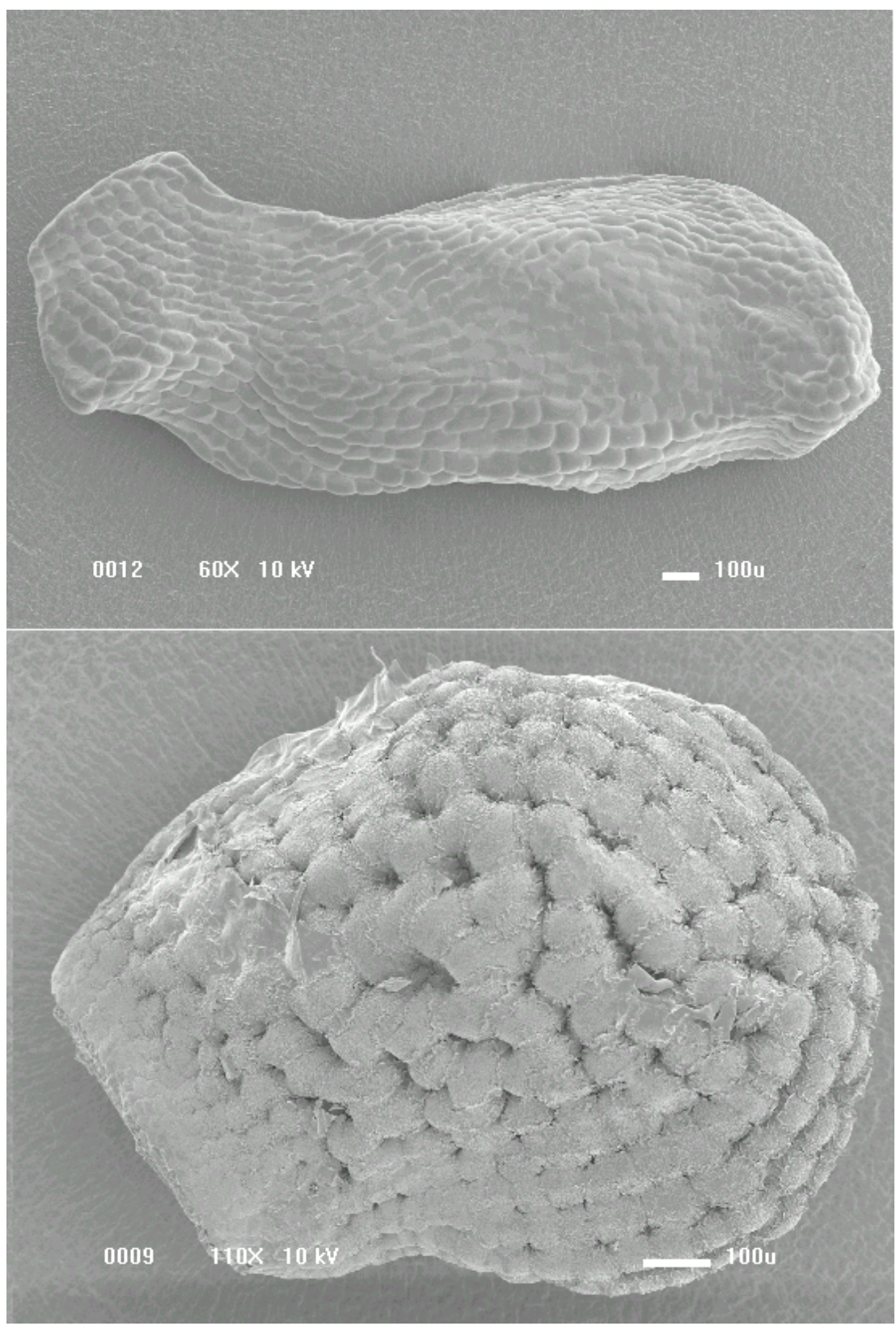


Os estudos que avaliaram a morfologia de cactos em diferentes estádios ontogenéticos, desde as sementes até as plântulas, passando pelas fases de germinação até o desenvolvimento inicial, foram publicados por Abud et al. com as três espécies de cactos colunares que apresentam as maiores distribuições geográficas na Caatinga: C. jamacaru ssp. jamacaru (Abud et al. 2013), Pilosocereus gounellei (F.A.C. Weber) Byles \& G.D. Rowley ssp. gounellei (Abud et al. 2012a) e Pilosocereus pachycladus F.Ritter ssp. pernambucoensis (F.Ritter) Zappi (Abud et al. 2010). Segundo Abud et al. (2010), as sementes de P. pachycladus ssp. pernambucoensis, espécie conhecida popularmente como facheiro, são estenospérmicas, exalbuminosas, com formato ligeiramente obovado-oblíqua. A testa é rugosa e de coloração preta. As sementes são campilótropas, exotestais, bitegumentadas. O embrião é cilíndrico e grande, sendo facilmente observado quando a semente está hidratada. Apresenta coloração branca e ocupa quase todo espaço da semente. Já o tecido de reserva é cotiledonar, de coloração branca e com consistência firme. Essas sementes de facheiro apresentam 1,55 mm de comprimento médio e, aproximadamente 96 horas após a semeadura, é observada a protrusão radicular, através da abertura do opérculo. Esta estrutura cresce rapidamente e, aos seis dias após a semeadura, já é observada uma plântula com comprimento médio de 1,98 $\mathrm{mm}$. Posteriormente, pode ser observado o desenvolvimento do hipocótilo que, após 10 dias de semeadura, encontra-se com aproximadamente $12 \mathrm{~mm}$ de comprimento, sendo possível observar também a presença de pelos radiculares nas raízes. Ainda, o desenvolvimento do epicótilo dessa espécie ocorre de forma muito lenta, com 60 dias após a semeadura e a presença de uma grande quantidade de espinhos pode ser observada a partir dos 90 dias de desenvolvimento (Abud et al. 2010). Esse padrão de desenvolvimento lento para a formação do epicótilo, bem como as mudanças morfológicas visualizadas ao longo do processo germinativo e de estabelecimento inicial das plântulas também é observado nas espécies C. jamacaru ssp. jamacaru (Abud et al. 2013) e P. gounellei ssp. gounellei (Abud et al. 2012a).

\section{Comportamento germinativo e fatores bióticos e abióticos que afetam a germinação}

Após a revisão bibliográfica, mais de 50 artigos sobre o comportamento germinativo e fatores bióticos e abióticos que afetam a germinação de sementes de cactos que ocorrem no Brasil foram consultados e suas informações foram agrupadas em cinco temas distintos que são apresentados abaixo.

\section{Tipos de reservas de sementes de cactos}

A informação sobre os tipos de reservas de sementes de cactos do Brasil ainda é pouquíssimo explorada na literatura atual. Um exemplo que pode ser dado é o estudo realizado por Alencar et al. (2012a) com sementes de C. jamacaru ssp. jamacaru, conhecido popularmente como mandacaru. Os lipídios representam o tipo de reserva mais abundante presente nas sementes de mandacaru. De acordo com os autores, cerca de 60\% da biomassa seca dos cotilédones dessa espécie é representada por lipídios, seguidos por proteínas e açúcares solúveis presentes em menor quantidade. Além disso, os lipídios são as principais reservas mobilizadas durante o processo germinativo, pois seus níveis são fortemente reduzidos após a germinação, indicando que as sementes de mandacaru utilizam essas reservas para germinar, sendo classificadas como oleaginosas (Alencar et al. 2012a). 


\section{Fotoblastismo}

Os resultados apresentados nesta revisão indicaram a ocorrência de dois comportamentos bem distintos em relação à resposta à luz durante a germinação de sementes de cactos do Brasil: (1) espécies que produzem sementes que germinam apenas na presença da luz e são consideradas fotoblásticas positivas (todas as espécies estudadas da subfamília Cactoideae) e (2) espécies que produzem sementes que também são capazes de germinar no escuro contínuo, sendo classificadas como fotoblásticas neutras ou afotoblásticas (todas as espécies estudadas das subfamílias Opuntioideae e Pereskioideae, veja Tabela 1). A luz é um dos sinais ambientais mais importantes que atuam no controle de diversas fases do ciclo de vida das plantas como, por exemplo, a germinação (Baskin \& Baskin 2014). A sensibilidade à luz durante a germinação de sementes é uma estratégia importante para prevenir a ocorrência de eventos germinativos em locais e épocas desfavoráveis ao estabelecimento das plântulas (Flores et al. 2011). O efeito da luz na germinação de sementes de representantes da família Cactaceae já foi avaliado em muitas espécies que ocorrem fora do Brasil e comportamentos similares aos descritos na presente revisão foram documentados em cactos que ocorrem em outros ecossistemas da América Central e da América do Norte (Rojas-Aréchiga \& Vázquez-Yanes 2000, Flores et al. 2006, 2011).

Os primeiros estudos que avaliaram o fotoblastismo das sementes de Cactaceae foram desenvolvidos nos Estados Unidos por Alcorn \& Kurtz Jr. no final da década de 1950, os quais indicaram a sensibilidade à luz do cacto colunar Carnegiea gigantea (Engelm.) Britton \& Rose (Alcorn \& Kurtz 1959). Respostas semelhantes foram observadas em todos os cactos colunares citados nesta revisão (Tabela 1), como os representantes dos gêneros Cereus Mill., Facheiroa Britton \& Rose e Pilosocereus Byles \& Rowley, os quais germinaram apenas na presença da luz. Após essa primeira publicação em 1959, alguns estudos foram desenvolvidos para caracterizar a resposta fotoblásticas de outras espécies de cactos em todo continente americano e o requerimento de luz para a germinação de sementes também foi relacionado aos atributos biológicos das espécies, tais como o tamanho das sementes e a perenialidade das plantas (veja revisão em Rojas-Aréchiga \& Vázquez-Yanes 2000). Então, em 1997, Rojas-Aréchiga et al. testaram a hipótese de que o fotoblastismo dos cactos estaria relacionado à forma de vida da espécie. De acordo com os autores, cactos globosos e em formato de barril sempre seriam fotoblásticos positivos e cactos colunares poderiam ser fotoblásticos positivos ou neutros. Esse padrão de resposta fotoblástica proposto por Rojas-Aréchiga et al. (1997) se deve às condições microclimáticas que prevalecem durante o desenvolvimento das sementes e promovem um efeito materno que gera diferentes requerimentos à luz (RojasAréchiga et al. 1997). Cactos globosos e em formato de barril produzem seus frutos mais próximos ao solo, onde a temperatura é relativamente maior. Por outro lado, a produção de frutos em cactos colunares ocorre nas extremidades dos cladódios, o que proporcionaria temperaturas mais amenas durante a produção das sementes (Rojas-Aréchiga et al. 1997). Assim, as diferenças de temperatura que as sementes são submetidas durante a sua produção determinaria, segundo os autores, o fotoblastismo das diferentes espécies de cactos.

Embora esse padrão de resposta fotoblástica proposto por Rojas-Aréchiga et al. (1997) tenha sido observado nos cactos brasileiros (todos os cactos globosos e colunares avaliados nesta revisão foram fotoblásticos positivos, veja as espécies na Tabela 1) e em diversos estudos com cactos nativos de outros países (veja todas as citações de espécies de cactos globosos e colunares de outros países em Meiado et al. 2016), Meiado et al. (2016) afirmaram que os atributos biológicos das espécies (e.g., forma de vida e tamanho de semente) não influenciam a resposta fotoblásticas dos cactos que ocorrem no Brasil. Essas afirmações não corroboram a hipótese proposta por 
Rojas-Aréchiga et al. (1997) que relacionaram a fotossensibilidade das sementes à forma de vida das espécies e sugerem que as respostas à luz dos cactos que ocorrem nos ecossistemas brasileiros não depende da sua forma de vida. Segundo Meiado et al. (2016), essas respostas podem estar relacionadas à origem filogenética, pois todas as espécies de uma mesma subfamília apresentaram o mesmo comportamento germinativo quando as sementes são submetidas à luz ou ao escuro contínuo. Tais resultados conflitantes sugerem que os padrões de respostas fotoblásticas propostos por Rojas-Aréchiga et al. (1997) possuem algumas limitações, as quais podem estar relacionadas ao fato de que estes foram propostos com base em dados de poucas espécies (3 cactos colunares e 4 cactos globosos) e nem todas as formas de vida que ocorrem na família Cactaceae foram avaliadas no referido estudo [i.e., articulados, cilíndricos, colunar, globoso, em forma de roseta, arbustos com folhas verdadeiras, segundo Anderson (2001)].

Como visto, a fotossensibilidade das sementes dos cactos vem sendo avaliada há muitos anos, porém, a maioria dos táxons estudados até então ( $>50 \%$ das espécies) ocorre exclusivamente no México (Flores et al. 2011). Embora o Brasil represente o país com a terceira maior diversidade de cactos do mundo, depois do México e do Chile (Taylor \& Zappi 2004), os estudos realizados com espécies brasileiras representavam, antes da compilação dos dados desta revisão, pouco mais de 5\% de todas as espécies avaliadas (Flores et al. 2011). Todos os estudos realizados até o momento confirmaram o comportamento apresentado nesta revisão, onde as espécies da subfamília Cactoideae, as quais representaram 85,1\% das espécies estudadas no Brasil, apresentaram fotoblastismo positivo, independentemente da forma de vida, do tamanho da semente, ou do ecossistema onde a espécie ocorre.

Como mencionado anteriormente, todas as espécies de cactos do Brasil estudadas até o momento que pertencem à subfamília Cactoideae são fotoblásticas positivas. Entretanto, alguns estudos apontaram representantes dessa subfamília como afotoblásticos ou fotoblásticos neutros. Esses resultados contraditórios podem estar relacionados à técnica escolhida para a avaliação das sementes submetidas ao escuro continuo que, na maioria das vezes, utiliza a luz verde de segurança. Embora Alencar et al. (2012b) tenham indicado o mandacaru como uma espécie fotoblásticas neutra, apresentando valores acima de $70 \%$ de germinação para sementes que foram mantidas no escuro contínuo e em temperaturas de $20^{\circ} \mathrm{C}$, é preciso ter cautela na interpretação da influência da luz na germinação das sementes dessa espécie. Quando sementes de mandacaru são mantidas constantemente no escuro, sem nenhuma avaliação periódica na luz verde de segurança, não é observada a germinação de nenhuma semente (Meiado 2012a, 2012b, Meiado et al. 2010, 2012b, 2016). Esses resultados indicam que a técnica utilizada para contagem da germinação de sementes no escuro não é segura para a espécie em questão e pode proporcionar a germinação das sementes que, na realidade, são fotoblásticas positivas e precisam de luz para germinar como todas as espécies da subfamília Cactoideae que ocorrem no Brasil.

\section{Influência da temperatura na germinação}

Segundo Rojas-Aréchiga \& Vázquez-Yanes (2000), a maioria das espécies de cactos responde positivamente a um amplo intervalo de temperatura durante a germinação $\left(17-34^{\circ} \mathrm{C}\right)$, com valores de temperatura ótima, frequentemente, em torno de $25^{\circ} \mathrm{C}$. Quando foi avaliado o efeito da temperatura na germinabilidade das sementes de C. jamacaru ssp. jamacaru, os resultados indicaram uma temperatura ideal para a germinação variando 
entre 25 e $30^{\circ} \mathrm{C}$ (Meiado et al. 2010, 2016). Porém, o tempo médio de germinação mostrou que a germinação do mandacaru foi mais rápida quando as sementes foram submetidas a $30^{\circ} \mathrm{C}$. Essa resposta pode ser favorável para a germinação da espécie, uma vez que, mesmo durante a estação chuvosa, a temperatura da interface do solo na Caatinga pode ser elevada ao longo do dia. Como visto, outros parâmetros além da germinabilidade (e.g., tempo médio de germinação e índice de sincronização da germinação) devem ser considerados na avaliação das respostas da germinação de sementes de cactos aos fatores ambientais (Meiado et al. 2010). Esses parâmetros podem contribuir, substancialmente, com o entendimento dos processos de germinação de sementes e recrutamento das plântulas no campo, os quais são influenciados por muitos fatores abióticos.

De acordo com Meiado et al. (2016), a temperatura também proporciona comportamentos germinativos diferenciados nas espécies de cactos do Brasil que já foram estudadas. Porém, a resposta positiva a uma maior amplitude térmica durante a germinação não tem nenhuma relação com a origem filogenética e, consequentemente, com o grupo taxonômico que o cacto pertence. Independente da origem filogenética, espécies cuja germinabilidade foi superior a 50\% e ocorreu em uma maior amplitude térmica são encontradas em áreas de Caatinga e Cerrado, locais onde a temperatura média anual é alta e pode ultrapassar os $40^{\circ} \mathrm{C}$ durante as estações mais quentes do ano. Por outro lado, sementes coletadas em áreas de Floresta Atlântica e Restinga, cujas temperaturas médias anuais são mais amenas, mostraram-se mais sensíveis às temperaturas extremas e à variação de temperatura. A diminuição da germinação de sementes das espécies brasileiras nas temperaturas extremas pode ter um significado ecológico, pois a sobrevivência das plântulas de cactos pode diminuir nessas temperaturas, impedindo o estabelecimento de plântulas em períodos do ano desfavoráveis (Meiado et al. 2010, 2012b, 2016).

Outro resultado observado por Meiado et al. (2016) que também sugere que o comportamento germinativo das sementes submetidas a diferentes temperaturas não está relacionado ao grupo taxonômico que a espécie pertence é a resposta germinativa diferencial entre subespécies. Embora tenha sido observada a mesma sensibilidade à luz, subespécies coletadas no mesmo ecossistema (Pilosocereus pentaedrophorus (J.F.Cels) Byles \& G.D.Rowley ssp. pentaedrophorus e Pilosocereus pentaedrophorus (J.F.Cels) Byles \& G.D.Rowley ssp. robustos Zappi - Mata Atlântica e Pilosocereus pachycladus F.Ritter ssp. pachycladus e P. pachycladus ssp. pernambucoensis Caatinga) apresentaram comportamento germinativo diferenciado quando as suas sementes foram submetidas a tratamentos de temperaturas extremas. Esse comportamento diferencial de resposta germinativa entre subespécies não é uma característica exclusiva das subespécies do gênero Pilosocereus. Resultados semelhantes também foram observados em subespécies do gênero Arthrocereus A. Berger [Arthrocereus melanurus (K.Schum.) Diers, P.J.Braun \& Esteves ssp. magnus N.P.Taylor \& Zappi, Arthrocereus melanurus (K.Schum.) Diers, P.J.Braun \& Esteves ssp. melanurus, Arthrocereus melanurus (K.Schum.) Diers, P.J.Braun \& Esteves ssp. odorus (Ritter) N.P.Taylor \& Zappi] que ocorrem em áreas de Campo Rupestre, em Minas Gerais (Cheib \& Garcia 2012). De acordo com Cheib \& Garcia (2012), embora as subespécies de A. melanurus tenham apresentado o mesmo comportamento germinativo nas temperaturas ótimas de germinação, a germinabilidade das sementes submetidas às temperaturas extremas foi significativamente diferente entre as subespécies avaliadas e $A$. melanurus ssp. melanurus apresentou maior germinabilidade e menor tempo de germinação nos tratamentos de 15 e $35^{\circ} \mathrm{C}$.

Com base nas informações apresentadas acima, onde é possível observar a influência do local de ocorrência da espécie no comportamento germinativo de sementes submetidas a diferentes temperaturas, acreditava-se, inicialmente, que a resposta positiva a uma maior amplitude térmica durante a germinação estaria relacionada 
a uma maior distribuição geográfica da espécie (Meiado 2012a, Meiado et al. 2012b). Tal suposição pode ser justificada pelo fato de que espécies de cactos com ampla distribuição geográfica como $P$. pachycladus ssp. pernambucoensis estão submetidas a diferentes condições ambientais durante a produção de sementes, ocasionando, assim, uma resposta positiva a uma maior amplitude térmica durante a germinação. Entretanto, espécies endêmicas e com distribuições pontuais também podem apresentar o mesmo padrão de resposta germinativa. Discocactus zehntneri Britton \& Rose ssp. petr-halfarii (Zachar) M.R.Santos \& M.C. Machado, uma espécies de cacto subgloboso que possui apenas uma única população na Caatinga, também apresenta uma resposta positiva a uma maior amplitude térmica durante a germinação, sendo observada uma germinabilidade similar entre todas as temperaturas avaliadas de 20 a $45^{\circ} \mathrm{C}$, confirmando, com isso, que a ampla distribuição geográfica de uma espécie não tem relação com a tolerância da espécie às temperaturas extremas (Nascimento 2014).

\section{Disponibilidade de água}

Além da luz e da temperatura, outros fatores ambientais, tais como a disponibilidade hídrica, pode afetar a germinação de sementes em ambientes áridos e semiáridos (Kigel 1995). De acordo com Kigel (1995), várias espécies do deserto são capazes de germinar em potencial relativamente baixo de água no solo, no entanto, a germinação diminui com a redução na disponibilidade hídrica. De acordo com Meiado et al. (2010), apesar de não ter sido observada germinação de semenes de C. jamacaru ssp. jamacaru no potencial de -1,0 MPa, foram observadas germinação de sementes em baixos potenciais hídricos, bem como uma redução significativa na germinabilidade das sementes com a redução da disponibilidade hídrica.

O mesmo padrão de resposta germinativa de sementes submetidas ao estresse hídrico também foi observado por Nascimento (2014) em sementes de espécies de Discocactus. Segundo Nascimento (2014), independente da espécie, a germinação das sementes em diferentes soluções de PEG 6000 foi observada em concentração de até -0,6 $\mathrm{MPa}$, sob luz branca, indicando a menor dependência da água no início do processo germinativo das espécies de Discocactus estudadas. No entanto, foi observado um decréscimo na germinabilidade dessas espécies à medida que se aumentava a concentração da solução de PEG 6000. Para a espécie D. babiensis, a redução da germinabilidade foi de $83,0 \pm 6,8 \%$ no tratamento controle para $7,0 \pm 3,8 \%$ na concentração $-0,6 \mathrm{MPa}$. Na espécie D. zehntneri ssp. zehntneri o decréscimo observado por Nascimento (2014) foi ainda maior, de 98,0 \pm 4,0\% (controle) para 4,0 \pm 4,6\% (-0,6 MPa), enquanto a espécie D. zehntneri ssp. petr-halfari apresentou uma germinação de $97,0 \pm 3,8 \%$ no tratamento controle, reduzindo a sua germinabilidade para 3,0 $\pm 3,8 \%$ quando as sementes foram submetidas a redução da disponibilidade hídrica.

A tolerância ao déficit hídrico também é alterada quando outro fator ambiental como, por exemplo, a temperatura atua em conjunto com a restrição da disponibilidade de água durante a germinação. Sementes de Pereskia grandifolia Haw. ssp. grandifolia apresentaram uma redução da tolerância ao déficit hídrico quando suas sementes foram submetidas a um aumento de temperatura de 25 para $30^{\circ} \mathrm{C}$ (Oliveira et al. 2017). De acordo com Oliveira et al. (2017), sementes de P. grandifolia ssp. grandifolia são capazes de germinar até potenciais de $-0,9005$ $\mathrm{MPa}$ quando submetidas a temperatura de $25^{\circ} \mathrm{C}$. Porém, a tolerância ao déficit hídrico reduz quando as sementes germinam em $30^{\circ} \mathrm{C}$, sendo observada a germinação até o potencial de $-0,7540 \mathrm{MPa}$. Como, no ambiente, a baixa disponibilidade de água está, geralmente, associada a períodos do ano com temperaturas elevadas, a tolerância ao déficit hídrico de sementes de algumas espécies de cactos observadas em condições de laboratório pode não 
ser a mesma observada em condições de campo.

Mais recentemente, Lima \& Meiado (2017) chamaram atenção para o fato de que os estudos realizados em condições controladas de laboratório que avaliam a influência de fatores abióticos sobre a germinação de espécies nativas e caracterizam sua tolerância e padrões de respostas germinativas devem ser considerados com cautela, uma vez que não demonstram a verdadeira tolerância aos estresses ambientas que sementes de espécies nativas apresentam no campo, após passarem por hidratação descontínua natural. De acordo com os autores, sementes de cactos como, por exemplo, da espécie Pilosocereus catingicola (Gürke) Byles \& Rowley ssp. salvadorensis (Werderm.) Zappi apresentam memória hídrica e aumentam a tolerância ao estresse hídrico após serem submetidas à hidratação descontínua. Além disso, a memória hídrica das sementes de cactos depende do clima e do microambiente onde as espécies estão estabelecidas, porque populações de uma mesma espécie que ocorrem em diferentes ecossistemas apresentaram diferentes respostas germinativas depois de passarem pelos ciclos de hidratação e desidratação e serem submetidas ao estresse, demonstrando que as características ambientais estão diretamente relacionadas à memória hídrica das sementes (Lima 2017; Lima \& Meiado 2017).

\section{Conclusão}

Como mencionado anteriormente, os estudos relacionados à morfofisiologia de sementes e plântulas de cactos que ocorrem no Brasil ainda são recentes e muitas espécies dessa família não foram avaliadas até o presente momento. A maioria das espécies estudadas depende da luz para germinar e a temperatura ideal de germinação de sementes está relacionada com o ecossistema onde a espécie ocorre, sendo observadas espécies na Caatinga com temperaturas ótimas de germinação acima de $30^{\circ} \mathrm{C}$, enquanto que espécies de matas úmidas, como a Mata Atlântica, tendem a apresentar um melhor desempenho germinativo em temperaturas entre $20 \mathrm{e}$ $25^{\circ} \mathrm{C}$. Finalmente, esta revisão mostrou que a família Cactaceae foi negligenciada por muito tempo nos estudos sobre o comportamento germinativo desenvolvidos no Brasil. Devido à importância ecológica e sociocultural das espécies dessa família e ao elevado número de espécies ameaçadas de extinção e que são comercializadas de forma ilegal, novos estudos sobre a temática abordada são incentivados, principalmente levando-se em consideração as espécies que nunca foram avaliadas e sobre as quais nada se sabe sobre seu comportamento germinativo. Tais estudos podem servir de base para projetos que visem à conservação ex situ, a criação de bancos de germoplasma, a produção de mudas, a recomposição de espécies e o enriquecimento de áreas degradadas, sendo importantíssimos para a preservação dos cactos em nosso país. 


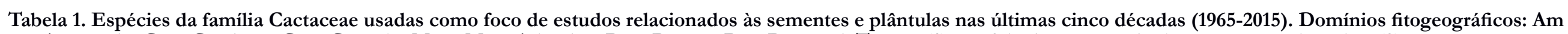

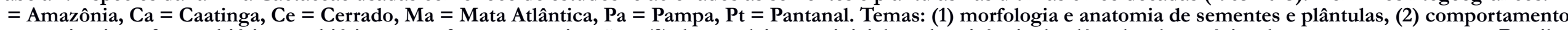

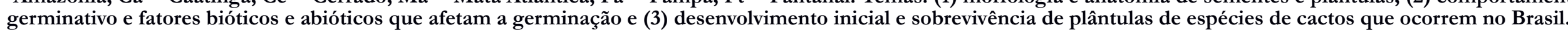

\begin{tabular}{|c|c|c|c|c|c|c|c|c|c|c|}
\hline \multirow{2}{*}{$\begin{array}{l}\text { SUBFAMÍLIA (nº gênero: } n^{\circ} \text { espécies) } \\
\text { Espécie }\end{array}$} & \multicolumn{6}{|c|}{ Domínio Fitogeográfico } & \multicolumn{3}{|c|}{ Tema } & \multirow{2}{*}{ Referências } \\
\hline & Am & $\mathbf{C a}$ & $\mathrm{Ce}$ & Ma & $\mathbf{P a}$ & Pt & 1 & 2 & 3 & \\
\hline \multicolumn{11}{|l|}{ CACTOIDEAE (19:61) } \\
\hline Arrojadoa rhodantha (Gürke) Britton \& Rose (NE) & & $\mathrm{X}$ & & & & & $\mathrm{X}$ & $\mathrm{X}$ & $\mathrm{X}$ & $37 ; 64 ; 71 ; 72$ \\
\hline Arthrocereus glaziovii (K.Schum.) N.P.Taylor \& Zappi (EN) & & & $\mathrm{X}$ & & & & & $\mathrm{X}$ & & $26 ; 27 ; 65$ \\
\hline Arthrocereus melanurus (K.Schum.) Diers et al. ssp. magnus N.P.Taylor \& Zappi (EN) & & & $\mathrm{X}$ & & & & & $\mathrm{X}$ & & $26 ; 27 ; 65$ \\
\hline Arthrocereus melanurus (K.Schum.) Diers et al. ssp. melanurus (EN) & & & $\mathrm{X}$ & & & & & $\mathrm{X}$ & & $26 ; 27 ; 65$ \\
\hline Arthrocereus melanurus (K.Schum.) Diers et al. ssp. odorus (Ritter) N.Taylor \& Zappi (EN) & & & $\mathrm{X}$ & & & & & $\mathrm{X}$ & & $26 ; 27 ; 65$ \\
\hline Cereus albicaulis (Britton \& Rose) Luetzelb. (NE) & & $\mathrm{X}$ & & & & & $\mathrm{X}$ & $\mathrm{X}$ & & $64 ; 71 ; 72 ; 76 ; 77$ \\
\hline Cereus fernambucensis Lem. ssp. fernambucensis (NE) & & & & $\mathrm{X}$ & & & $\mathrm{X}$ & $\mathrm{X}$ & & $\begin{array}{l}14 ; 15 ; 39 ; 64 ; 65 \\
71 ; 76 ; 77 ; 94 ; 104\end{array}$ \\
\hline Cereus hildmannianus K.Schum. ssp. hildmannianus (NE) & & & & $\mathrm{X}$ & & & & $\mathrm{X}$ & $\mathrm{X}$ & $13 ; 22 ; 24 ; 45$ \\
\hline Cereus jamacaru DC. ssp. calcirupicola (F.Ritter) N.P.Taylor \& Zappi (NE) & & & $\mathrm{X}$ & & & & $\mathrm{X}$ & & & \\
\hline Cereus jamacaru DC. ssp. jamacaru (NE) & & $\mathrm{X}$ & & & & & $\mathrm{X}$ & $\mathrm{X}$ & $\mathrm{X}$ & $\begin{array}{l}2 ; 6 ; 9 ; 10 ; 11 ; 21 ; \\
25 ; 29 ; 30 ; 38 ; 39 ; \\
43 ; 64 ; 65 ; 69 ; 71 ; \\
72 ; 76 ; 77 ; 81 ; 85 ; \\
90 ; 91 ; 95 ; 108\end{array}$ \\
\hline Cipocereus minensis (Werderm.) Ritter ssp. leiocarpus N.P.Taylor \& Zappi (LC) & & & $\mathrm{X}$ & & & & & $\mathrm{X}$ & $\mathrm{X}$ & 57 \\
\hline Cipocereus minensis (Werderm.) Ritter ssp. minensis (NE) & & & $\mathrm{X}$ & $\mathrm{X}$ & & & & $\mathrm{X}$ & & 86 \\
\hline Coleocephalocereus fluminensis (Miq.) Backeb. (NE) & & & & $\mathrm{X}$ & & & $\mathrm{X}$ & $\mathrm{X}$ & & $14 ; 15 ; 65 ; 92$ \\
\hline Coleocephalocereus goebelianus (Vaupel) Buining (NE) & & $\mathrm{X}$ & & & & & $\mathrm{X}$ & & & 17 \\
\hline Discocactus bahiensis Britton \& Rose (VU) & & $\mathrm{X}$ & & & & & $\mathrm{X}$ & $\mathrm{X}$ & $\mathrm{X}$ & $64 ; 71 ; 72 ; 73 ; 74$ \\
\hline Discocactus placentiformis (Lehm.) K.Schum. (NT) & & & $\mathrm{X}$ & & & & & $\mathrm{X}$ & & 100 \\
\hline Discocactus pseudoinsignis N.P.Taylor \& Zappi (CR) & & & $\mathrm{X}$ & & & & & $\mathrm{X}$ & & 100 \\
\hline $\begin{array}{l}\text { Discocactus zehntneri Britton \& Rose ssp. boomianus (Buining \& Brederoo) N.P.Taylor \& } \\
\text { Zappi (NE) }\end{array}$ & & $X$ & & & & & & $X$ & & 72 \\
\hline $\begin{array}{l}\text { Discocactus zehntneri Britton \& Rose ssp. petr-halfarii (Zachar) M.R.Santos \& M.C. Machado } \\
\text { (CR) }\end{array}$ & & $X$ & & & & & & $X$ & $X$ & 73 \\
\hline Discocactus zehntneri Britton \& Rose ssp. zehntneri (NE) & & $X$ & & & & & $X$ & $X$ & $X$ & $\begin{array}{l}58 ; 59 ; 64 ; 65 ; 71 ; \\
72 ; 73 ; 108\end{array}$ \\
\hline
\end{tabular}

Categorias de ameaça: $\mathrm{NE}=$ espécie não avaliada quanto à ameaça de extinção. $\mathrm{EN}=$ em perigo de extinção. VU = vulnerável. CR $=$ criticamente em perigo de extinção. $\mathrm{LC}=$ pouco preocupante. $\mathrm{DD}=$ deficiente de dados. $\mathrm{NT}=$ quase ameaçada de extinção. $*$ espécie naturalizada. 
Tabela 1. Continuação.

\begin{tabular}{|c|c|c|c|c|c|c|c|c|c|c|}
\hline \multirow{2}{*}{$\begin{array}{l}\text { SUBFAMÍLIA (nº gênero: } n^{\circ} \text { espécies) } \\
\text { Espécie }\end{array}$} & \multicolumn{6}{|c|}{ Domínio Fitogeográfico } & \multicolumn{3}{|c|}{ Tema } & \multirow[t]{2}{*}{ Referências } \\
\hline & Am & $\mathbf{C a}$ & $\mathrm{Ce}$ & Ma & $\mathbf{P a}$ & $\mathbf{P t}$ & 1 & 2 & 3 & \\
\hline Echinopsis rhodotricha K.Schum. (NE) & & & & & & $\mathrm{X}$ & $\mathrm{X}$ & & & $7 ; 8$ \\
\hline Epiphyllum phyllanthus (L.) Haw. (LC) & & & & $\mathrm{X}$ & & & & $\mathrm{X}$ & $\mathrm{X}$ & $12 ; 101 ; 107$ \\
\hline Facheiroa squamosa (Gürke) P.J.Braun \& E.Esteves Pereira (NE) & & $\mathrm{X}$ & & & & & $\mathrm{X}$ & $\mathrm{X}$ & & $64 ; 71 ; 72$ \\
\hline Harrisia adscendens (Gürke) Britton \& Rose (NE) & & $\mathrm{X}$ & & & & & $\mathrm{X}$ & $\mathrm{X}$ & & $64 ; 71 ; 72 ; 78$ \\
\hline Hylocereus megalanthus (K.Schum. ex Vaupel) Ralf Bauer (NE)* & & & & & & & & $\mathrm{X}$ & & $51 ; 52 ; 56$ \\
\hline Hylocereus polyrhizus (Weber) Britton \& Rose (NE)* & & & & & & & & $\mathrm{X}$ & & $51 ; 52 ; 56$ \\
\hline Hylocereus setaceus (Salm-Dyck) R.Bauer (LC) & & & & $\mathrm{X}$ & & & $\mathrm{X}$ & $\mathrm{X}$ & & $65 ; 102 ; 103$ \\
\hline Hylocereus undatus (Haw.) Britton \& Rose (NE)* & & & & $\mathrm{X}$ & & & & $\mathrm{X}$ & & $16 ; 41 ; 56 ; 60 ; 80 ; 97$ \\
\hline Lepismium cruciforme (Vell.) Miq. (LC) & & & & $\mathrm{X}$ & & & $\mathrm{X}$ & & & 96 \\
\hline Melocactus azureus Buining \& Brederoo (EN) & & $\mathrm{X}$ & & & & & & $\mathrm{X}$ & & 19 \\
\hline Melocactus bahiensis (Britton \& Rose) Luetzelb. ssp. bahiensis (NE) & & $\mathrm{X}$ & & & & & $\mathrm{X}$ & $\mathrm{X}$ & $\mathrm{X}$ & $\begin{array}{l}25 ; 36 ; 55 ; 64 ; 65 ; \\
71 ; 72\end{array}$ \\
\hline Melocactus concinnus Buining \& Brederoo (NE) & & $\mathrm{X}$ & $\mathrm{X}$ & & & & & $\mathrm{X}$ & & 108 \\
\hline Melocactus conoideus Buining \& Brederoo (CR) & & $\mathrm{X}$ & & & & & $\mathrm{X}$ & $\mathrm{X}$ & & $17 ; 65 ; 83 ; 84$ \\
\hline Melocactus deinacanthus Buining \& Brederoo (CR) & & $\mathrm{X}$ & & & & & & $\mathrm{X}$ & $\mathrm{X}$ & 93 \\
\hline Melocactus ernestii Vaupel ssp. ernestii (NE) & & $\mathrm{X}$ & & & & & $\mathrm{X}$ & $\mathrm{X}$ & & $1 ; 18 ; 31 ; 32 ; 64 ; 71 ; 72$ \\
\hline Melocactus glaucescens Buining \& Brederoo (EN) & & $\mathrm{X}$ & & & & & & $\mathrm{X}$ & $\mathrm{X}$ & $32 ; 72 ; 88 ; 89$ \\
\hline Melocactus oreas Miq. ssp. cremnophilus (Buining \& Brederoo) P.J.Braun (NE) & & $\mathrm{X}$ & & & & & $\mathrm{X}$ & $\mathrm{X}$ & & $64 ; 71 ; 72$ \\
\hline Melocactus paucispinus Heimen \& R.J.Paul (EN) & & $\mathrm{X}$ & & & & & $\mathrm{X}$ & $\mathrm{X}$ & & $1 ; 72 ; 108$ \\
\hline Melocactus sergipensis N.P.Taylor \& M.V.Meiado (CR) & & $\mathrm{X}$ & & & & & $\mathrm{X}$ & $\mathrm{X}$ & $\mathrm{X}$ & $67 ; 70 ; 94$ \\
\hline Melocactus violaceus Pfeiff. ssp. margaritaceus N.P.Taylor (VU) & & & & $\mathrm{X}$ & & & & $\mathrm{X}$ & & 94 \\
\hline Melocactus violaceus Pfeiff. ssp. violaceus (VU) & & & & $\mathrm{X}$ & & & $\mathrm{X}$ & $\mathrm{X}$ & & $34 ; 40 ; 64 ; 71 ; 109$ \\
\hline Melocactus zehntneri (Britton \& Rose) Luetzelb. (NE) & & $\mathrm{X}$ & & & & & $\mathrm{X}$ & $\mathrm{X}$ & & $\begin{array}{l}18 ; 20 ; 29 ; 47 ; 64 ; \\
71 ; 72 ; 94\end{array}$ \\
\hline Melocactus x albicephalus Buining \& Brederoo (NE) & & $\mathrm{X}$ & & & & & & $\mathrm{X}$ & & $32 ; 108$ \\
\hline Micranthocereus auriazureus Buining \& Brederoo (EN) & & & $\mathrm{X}$ & & & & $\mathrm{X}$ & & & 17 \\
\hline Micranthocereus albicephalus (Buining \& Brederoo) F.Ritter (EN) & & & $\mathrm{X}$ & & & & $\mathrm{X}$ & & & 17 \\
\hline Micranthocereus dolichospermaticus (Buining \& Brederoo) F.Ritter (EN) & & & $\mathrm{X}$ & & & & $\mathrm{X}$ & & & 17 \\
\hline Micranthocereus estevesii (Buining \& Brederoo) F.Ritter (NE) & & & $\mathrm{X}$ & & & & $\mathrm{X}$ & & & 17 \\
\hline Micranthocereus flaviflorus Buining \& Brederoo (NE) & & $\mathrm{X}$ & & & & & $\mathrm{X}$ & $\mathrm{X}$ & & $17 ; 28 ; 72 ; 108$ \\
\hline Micranthocereus polyanthus (Werderm.) Backeb. ssp. alvini M.Machado \& Hofacker (NE) & & $\mathrm{X}$ & & & & & & $\mathrm{X}$ & & 28 \\
\hline Micranthocereus polyanthus (Werderm.) Backeb. ssp. polyanthus (NE) & & & $\mathrm{X}$ & & & & $\mathrm{X}$ & & & 17 \\
\hline
\end{tabular}


Tabela 1. Continuação.

\begin{tabular}{|c|c|c|c|c|c|c|c|c|c|c|}
\hline \multirow{2}{*}{$\begin{array}{l}\text { SUBFAMÍLIA (nº gênero: } n^{\circ} \text { espécies) } \\
\text { Espécie }\end{array}$} & \multicolumn{6}{|c|}{ Domínio Fitogeográfico } & \multicolumn{3}{|c|}{ Tema } & \multirow{2}{*}{ Referências } \\
\hline & Am & $\mathbf{C a}$ & $\mathrm{Ce}$ & Ma & $\mathbf{P a}$ & $\mathbf{P t}$ & 1 & 2 & 3 & \\
\hline Micranthocereus purpureus (Gürke) F.Ritter (NE) & & & $\mathrm{X}$ & & & & $\mathrm{X}$ & & & 17 \\
\hline Micranthocereus streckeri Van Heek \& Van Criek. (CR) & & & $\mathrm{X}$ & & & & $\mathrm{X}$ & & & 17 \\
\hline Micranthocereus violaciflorus Buining (EN) & & & $\mathrm{X}$ & & & & $\mathrm{X}$ & & & 17 \\
\hline Parodia leninghausii (K.Schum.) F.H.Brandt. (DD) & & & & & $\mathrm{X}$ & & & $\mathrm{X}$ & & $64 ; 110$ \\
\hline Pilosocereus arrabidae (Lem.) Byles \& G.D.Rowley (LC) & & & & $\mathrm{X}$ & & & & $\mathrm{X}$ & & $14 ; 15 ; 61 ; 62 ; 65$ \\
\hline Pilosocereus aurisetus (Werderm.) Byles \& G.D.Rowley ssp. aurisetus (NE) & & & $\mathrm{X}$ & & & & $\mathrm{X}$ & $\mathrm{X}$ & $\mathrm{X}$ & $17 ; 86 ; 87$ \\
\hline Pilosocereus catingicola (Gürke) Byles \& Rowley ssp. salvadorensis (Werderm.) Zappi (NE) & & & & $\mathrm{X}$ & & & $X$ & $\mathrm{X}$ & $\mathrm{X}$ & $\begin{array}{l}48 ; 49 ; 50 ; 63 ; 64 \\
65 ; 68 ; 71 ; 72 ; 82 ; 94\end{array}$ \\
\hline Pilosocereus chrysostele (Vaupel) Byles \& G.D.Rowley (NE) & & $\mathrm{X}$ & & & & & $\mathrm{X}$ & $\mathrm{X}$ & & $64 ; 72 ; 76 ; 77$ \\
\hline Pilosocereus gounellei (F.A.C.Weber) Byles \& Rowley ssp. gounellei (NE) & & $\mathrm{X}$ & & & & & $X$ & $X$ & $X$ & $\begin{array}{l}2 ; 3 ; 5 ; 17 ; 25 ; 33 ; 42 ; 5 \\
8 ; 59 ; 64 ; 65 ; 71 ; 72 ; 7 \\
6 ; 77 ; 94 ; 98 ; 99 ; 108\end{array}$ \\
\hline $\begin{array}{l}\text { Pilosocereus gounellei (F.A.C.Weber) Byles \& Rowley ssp. zehntneri (Britton \& Rose) Zappi } \\
\text { (NE) }\end{array}$ & & & $\mathrm{X}$ & & & & $\mathrm{X}$ & & & 每, \\
\hline Pilosocereus pachycladus F.Ritter ssp. pachycladus (NE) & & $\mathrm{X}$ & & & & & X & $\mathrm{X}$ & & $19 ; 64 ; 71 ; 72 ; 76 ; 77$ \\
\hline Pilosocereus pachycladus F.Ritter ssp. pernambucoensis (F.Ritter) Zappi (NE) & & $X$ & & & & & $\mathrm{X}$ & $\mathrm{X}$ & $\mathrm{X}$ & $\begin{array}{l}4 ; 25 ; 29 ; 64 ; 65 ; 71 ; \\
72 ; 76 ; 77\end{array}$ \\
\hline Pilosocereus pentaedrophorus (J.F.Cels) Byles \& G.D.Rowley ssp. pentaedrophorus (NE) & & & & $\mathrm{X}$ & & & $\mathrm{X}$ & $\mathrm{X}$ & & $64 ; 71$ \\
\hline Pilosocereus pentaedrophorus (J.F.Cels) Byles \& G.D.Rowley ssp. robustus Zappi (NE) & & & & $\mathrm{X}$ & & & $\mathrm{X}$ & $\mathrm{X}$ & & $64 ; 71$ \\
\hline Pilosocereus piauhyensis (Gürke) Byles \& G.D.Rowley (LC) & & $\mathrm{X}$ & & & & & $\mathrm{X}$ & $\mathrm{X}$ & & $64 ; 71 ; 72$ \\
\hline Pilosocereus tuberculatus (Werderm.) Byles \& G.D.Rowley (NE) & & $\mathrm{X}$ & & & & & $\mathrm{X}$ & $\mathrm{X}$ & & $64 ; 71 ; 72 ; 76 ; 77$ \\
\hline Pilosocereus ulei (K.Schum.) Byles \& G.D.Rowley (NE) & & & & $\mathrm{X}$ & & & & $\mathrm{X}$ & & $14 ; 15 ; 65$ \\
\hline Rhipsalis cereuscula Haw. (NE) & & & & $\mathrm{X}$ & & & $\mathrm{X}$ & & & 96 \\
\hline Rhipsalis floccosa Salm-Dyck ex Pfeiff. ssp. floccosa (NE) & & & & $\mathrm{X}$ & & & & $\mathrm{X}$ & $\mathrm{X}$ & 53 \\
\hline Rhipsalis floccosa Salm-Dyck ex Pfeiff. ssp. hohenauensis (Ritter) Barth. \& N.P.Taylor (NE) & & & & $\mathrm{X}$ & & & $\mathrm{X}$ & & & 96 \\
\hline Rhipsalis pilocarpa Loefgr. (NT) & & & & $\mathrm{X}$ & & & & $\mathrm{X}$ & $\mathrm{X}$ & 53 \\
\hline Schlumbergera truncata (Haw.) Moran (LC) & & & & $X$ & & & & $\mathrm{X}$ & $\mathrm{X}$ & $46 ; 54 ; 65$ \\
\hline Stephanocereus luetzelburgii (Vaupel) N.P.Taylor \& Eggli (LC) & & $\mathrm{X}$ & & & & & & $\mathrm{X}$ & $\mathrm{X}$ & $58 ; 59$ \\
\hline \multicolumn{11}{|l|}{ OPUNTIOIDEAE (4:7) } \\
\hline Brasiliopuntia brasiliensis (Willd.) A.Berger (LC) & & & & $\mathrm{X}$ & & & $\mathrm{X}$ & $\mathrm{X}$ & & $64 ; 71 ; 72$ \\
\hline Nopalea cochenillifera (L.) Salm-Dyck (NE)* & & & & & & & & $\mathrm{X}$ & & 23 \\
\hline Opuntia ficus-indica Mill. (NE)* & & & & & & & & $\mathrm{X}$ & & 105 \\
\hline
\end{tabular}


Tabela 1. Continuação.

\begin{tabular}{|c|c|c|c|c|c|c|c|c|c|c|}
\hline \multirow{2}{*}{$\begin{array}{l}\text { SUBFAMÍLIA (nº gênero: } n^{\circ} \text { espécies) } \\
\text { Espécie }\end{array}$} & \multicolumn{6}{|c|}{ Domínio Fitogeográfico } & \multicolumn{3}{|c|}{ Tema } & \multirow[b]{2}{*}{ Referências } \\
\hline & Am & $\mathbf{C a}$ & $\mathrm{Ce}$ & Ma & $\mathbf{P a}$ & $\mathbf{P t}$ & 1 & 2 & 3 & \\
\hline Tacinga funalis Britton \& Rose (DD) & & $\mathrm{X}$ & & & & & $\mathrm{X}$ & $\mathrm{X}$ & & $64 ; 71 ; 72$ \\
\hline Tacinga inamoena (K.Schum.) N.P.Taylor \& Stuppy (DD) & & $\mathrm{X}$ & & & & & $\mathrm{X}$ & $\mathrm{X}$ & & $29 ; 44 ; 64 ; 71 ; 72 ; 75$ \\
\hline Tacinga palmadora (Britton \& Rose) N.P.Taylor \& Stuppy (LC) & & $\mathrm{X}$ & & & & & $\mathrm{X}$ & $\mathrm{X}$ & $\mathrm{X}$ & $64 ; 66 ; 71 ; 72$ \\
\hline Tacinga saxatilis (F.Ritter) N.P.Taylor \& Stuppy ssp. saxatilis (NE) & & $\mathrm{X}$ & & & & & $\mathrm{X}$ & $\mathrm{X}$ & & $64 ; 71$ \\
\hline \multicolumn{11}{|l|}{ PERESKIOIDEAE $(1: 4)$} \\
\hline Pereskia aculeata Mill. (LC) & & $\mathrm{X}$ & & & & & $\mathrm{X}$ & $\mathrm{X}$ & & $\begin{array}{l}35 ; 64 ; 65 ; 71 ; 72 ; \\
106\end{array}$ \\
\hline Pereskia bahiensis Gürke (LC) & & $\mathrm{X}$ & & & & & $\mathrm{X}$ & $\mathrm{X}$ & & $64 ; 71 ; 72$ \\
\hline Pereskia grandifolia Haw. ssp. grandifolia (NE) & & & & $\mathrm{X}$ & & & $\mathrm{X}$ & $\mathrm{X}$ & & $64 ; 71 ; 79 ; 106$ \\
\hline Pereskia sacharosa Griseb. (NE) & & & & & & $\mathrm{X}$ & & & $\mathrm{X}$ & $7 ; 8$ \\
\hline TOTAL & 0 & 37 & 19 & 21 & 1 & 2 & 53 & 68 & 21 & \\
\hline
\end{tabular}

1) Abreu (2008); 2) Abud (2010); 3) Abud et al. (2012a); 4) Abud et al. (2010); 5) Abud et al. (2012b); 6) Abud et al. (2013); 7) Alcântara (2008); 8) Alcântara et al. (2008); 9) Alencar

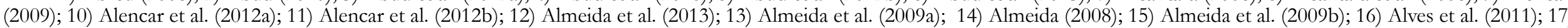
Aona (2003); 18) Assis et al. (2015); 19) Bárbara et al. (2015); 20) Bravo Filho (2014); 21) Brito et al. (2010); 22) Carvalho et al. (2008); 23) Castro et al. (2011); 24) Cavalcanti e Resende

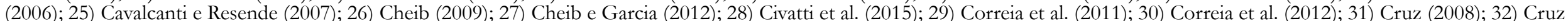

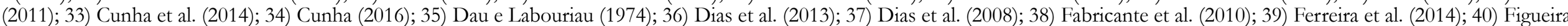

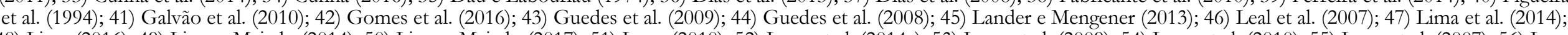

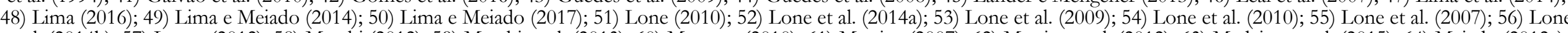

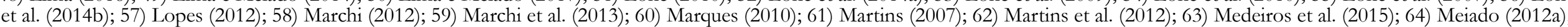

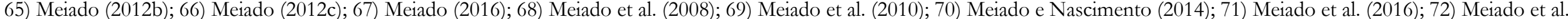

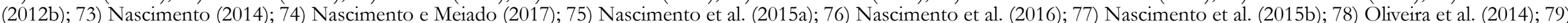

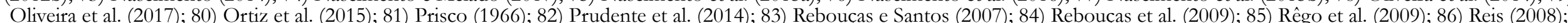

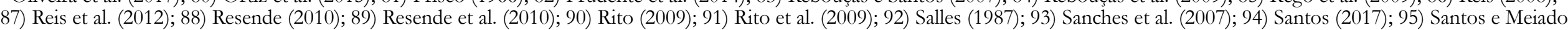

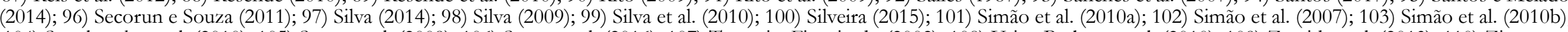

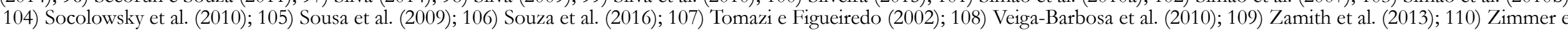
Büttner (1982) 


\section{REFERÊNCIAS}

Abreu DDS. 2008. Germinação e morfo-anatomia do desenvolvimento em Melocactus ernestii Vaupel e $M$. paucispinus Heimen \& R.J. Paul (Cactaceae). Dissertação de Mestrado. São Paulo: Universidade de São Paulo.

Adub HF. 2010. Germinação, morfologia e armazenamento de sementes de mandacaru (Cereus jamacaru DC.) e xique-xique (Pilosocereus gounellei (F.A.C. Weber) Byles \& G.D.Rowley). Dissertação de Mestrado. Fortaleza: Universidade Federal do Ceará.

Adub HF, Gonçalves NR, Pereira MS, Pereira DS, Reis RGE e Bezerra AME. 2012a. Germination and morphological characterization of the fruits, seeds, and seedlings of Pilosocereus gounellei. Brazilian Journal of Botany, 35(1): 11-16.

Adub HF, Gonçalves NR, Reis RGE, Pereira DS e Bezerra AME. 2010. Germinação e expressão morfológica de frutos, sementes e plântulas de Pilosocereus pachycladus Ritter. Revista Ciência Agronômica, 41(3): 468-474.

Abud HF, Pereira DS, Gonçalves NR, Pereira MS e Bezerra AME. 2012b. Armazenamento de sementes de xique-xique. Revista Brasileira de Sementes, 34(3): 473-479.

Adub HF, Pereira MS, Gonçalves NR, Pereira DS e Bezerra AME. 2013. Germination and morphology of fruits, seeds and plants of Cereus jamacaru DC. Journal of Seed Science, 35(3): 310-315.

Alcântara NV. 2008. Caracterização morfológica de frutos, sementes, germinação e desenvolvimento inicial de Echinopsis rhodotricha K. Schum. e desenvolvimento de Pereskia sacharosa Griseb. do Chaco de Porto Murtinho/MS. Campo Grande: Universidade Federal do Mato Grosso do Sul.

Alcântara NV, Menegucci ZRH, Scremin-Dias E e Garcia JS. 2008. Germinação, caracterização morfológica de frutos, sementes e desenvolvimento inicial de Cactaceae do Chaco de Porto Murtinho/MS. GeoPantanal, $1(1): 12-21$.

Alcorn SM e Kurtz Jr. EB. 1959. Some factors affecting the germination on the saguaro cactus (Carnegiea gigantea). American Journal of Botany, 46(7): 526-529.

Alencar NLM. 2009. Fisiologia, bioquímica e morfologia da germinação de mandacaru (Cereus jamacaru DC.). Dissertação de Mestrado. Fortaleza: Universidade Federal do Ceará.

Alencar NLM, Gomes-Filho E e Innecco R. 2012a. Cereus jamacaru seed germination and initial seedling establishment as a function of light and temperature condition. Scientia Agricola, 69(1): 70-74.

Alencar NLM, Innecco R, Gomes-Filho E, Gallão MI, Alvarez-Pizarro JC, Prisco JT e Oliveira AB. 2012b. Seed reserve composition and mobilization during germination and early seedling establishment of Cereus jamacaru DC. ssp. jamacaru (Cactaceae). Anais da Academia Brasileira de Ciências, 84(3): 823-832.

Almeida OJG, Paoli AAS, Souza LA e Cota-Sánches JH. 2013. Seedling morphology and development in the epiphytic cactus Epiphyllum phyllanthus (L.) Haw. (Cactaceae: Hylocereeae). Journal of the Torrey Botanical 
Society, 140(2): 196-214.

Almeida OJG, Souza LA e Moscheta IS. 2009a. Morfo-anatomia de plântulas de indivíduos somaclones de Cereus bildmannianus Schumann (Cactaceae). Boletín de la Sociedad Latinoamericana y del Caribe de Cactáceas y otras Suculentas, 6(3): 29-35.

Almeida TMH. 2008. Características físicas, germinação e conservação de sementes de cactáceas nativas da costa fluminense. Dissertação de Mestrado. Seropédica: Universidade Federal Rural do Rio de Janeiro.

Almeida TMH, Andrade ACS e Lopes HM. 2009b. Brazilian cacti seed germination under different temperature and substrate conditions. Seed Science \& Technology, 37(2): 474-479.

Alves CZ, Godoy AR e Corrêa LS. 2011. Adequação da metodologia para o teste de germinação de sementes de pitaia vermelha. Ciência Rural, 41(5): 779-784.

Anderson EF. 2001. The cactus family. Portland: Timber Press, Inc.

Aona LYS. 2003. Caracterização e delimitação do gênero Micranthocereus Backeb. (Cactaceae) baseadas em caracteres morfológicos e moleculares. Dissertação de Mestrado. Campinas: Universidade Estadual de Campinas.

Assis JGA, Pérez-García F e Gonzáles-Benito ME. 2015. Tetrazolium test for seed viability and germinability of Melocactus ernestii Vaupel subsp. ernestii and Melocactus zehntneri (Britton \& Rose) Luetzelb. (Cactaceae). Gaia Scientia, 9(2): 15-16.

Bárbara EPS, Silva AA, Souza MMOR, Gurgel ZER, Marchi MNG e Bellintani MC. 2015. Germinação e criopreservação de sementes de cactos nativos da Bahia. Gaia Scientia, 9(2): 91-96.

Baskin CC e Baskin JM. 2014. Seeds: ecology, biogeography, and evolution of dormancy and germination. San Diego: Academic Press.

Bravo Filho ES. 2014. Diversidade, etnobotânica e propagação de cabeça-de-frade (Melocactus Link \& Otto - Cactaceae) no Estado de Sergipe. Dissertação de Mestrado. São Cristóvão: Universidade Federal de Sergipe.

Brito NM, Nascimento LC, Coelho MSE e Félix LP. 2010. Efeitos de óleos essenciais na germinação de sementes de Cereus jamacaru. Revista Brasileira de Ciências Agrárias, 5(2): 207-211.

Carvalho VM, Mangolin CA e Machado MFPS. 2008. Seed germination of the Cereus perwvianus Mill. (Cactaceae) somaclones follows a relatively simple protocol. Seed Science \& Technology, 36(3): 595-600.

Castro JP, Araújo ER, Rêgo MM e Rêgo ER. 2011. In vitro germination and disinfestation of sweet cactus (Nopalea cochenillifera (L.) Salm-Dyck). Acta Scientiarum. Agronomy, 33(3): 509-512.

Cavalcanti NB e Resende GM. 2006. Efeitos de diferentes substratos no desenvolvimento de mandacaru sem espinhos (Cereus hildemannianus K.Schum). Revista Caatinga, 19(3): 255-260. 
Cavalcanti NB e Resende GM. 2007. Efeitos de diferentes substratos no desenvolvimento de mandacaru (Cereus jamacaru P.DC.), facheiro (Pilosocereus pachycladus Ritter), xique-xique (Pilosocereus gounellei (A. Weber ex K.Schum.) Bly. ex Rowl.) e coroa-de-frade (Melocactus bahiensis Britton \& Rose). Revista Caatinga, 20(1): 28-35.

Cheib AL. 2009. Ecologia da germinação e potencial para a formação de banco de sementes de espécies de Arthrocereus A.Berger (Cactaceae) endêmicas dos campos rupestres de Minas Gerais, Brasil. Dissertação de Mestrado. Belo Horizonte: Universidade Federal de Minas Gerais.

Cheib AL e Garcia QS. 2012. Longevity and germination ecology of seeds of endemic Cactaceae species from high-altitude sites in South-eastern Brazil. Seed Science Research, 22(1): 45-53.

Civatti LM, Marchi MNG e Bellintani MC. 2015. Conservação de sementes de cactos com potencial ornamental armazenadas sob diferentes condições de umidade e temperatura. Gaia Scientia, 9(2): 17-26.

Correia D, Nascimento EHS, Araújo JDM, Anselmo GC e Coelho PJA. 2011. Germinação de sementes de cactáceas in vitro. Comunicado Técnico 181. Fortaleza: Embrapa Agroindústria Tropical.

Correia D, Silva IC, Nascimento EHS e Morais JPS. 2012. Produção de mudas de mandacaru. Circular Técnica 39. Fortaleza: Embrapa Agroindústria Tropical.

Cruz BM. 2008. Avaliação da influência do armazenamento na germinação das sementes de Melocactus ernestii Vaupel. Monografia. Salvador: Centro Universitário Jorge Amado.

Cruz BM. 2011. Estudos de longevidade e germinação em sementes de espécies do gênero Melocactus (Cactaceae) de Morro do Chapéu, Chapada Diamantina, Bahia. Dissertação de Mestrado. Feira de Santana: Universidade Estadual de Feira de Santana.

Cunha MC, Lima AR, Nascimento AMJ e Meiado MV. 2014. Análise do potencial germinativo e caracterização morfométrica das sementes de xique-xique [Pilosocereus gounellei (F.A.C. Weber) Byles \& Rowley subsp. gounellei Cactaceae]. Informativo ABRATES, 24(3): 97.

Cunha MLC. 2016. Germinação de sementes de coroa-de-frade (Melocactus violaceus) visando à produção de mudas. Monografia. Natal: Universidade Federal do Rio Grande do Norte.

Dau L e Labouriau LG. 1974. Temperature control of seed germination in Pereskia aculeata Mill. Anais da Academia Brasileira de Ciências, 46(2): 311-322.

Dias MM, Nietsche S, Nunes CF, Matrangolo CAR e Pereira MCT. 2013. Reguladores de crescimento na emergência e desenvolvimento in vitro de Melocactus bahiensis. Tecnologia \& Ciência Agropecuária, 7(1): 7-11.

Dias MM, Nietsche S, Pereira MCT e Matrangolo CAR. 2008. Emergência e desenvolvimento da cactácea rabode-raposa (Arrojadoa spp.) em diferentes meios de cultura e recipientes. Revista Ceres, 55(2): 117-123.

Fabricante JR, Bezerra FTC, Souza VC, Feitosa SS, Andrade LA e Alves EU. 2010. Influência de temperatura e substrato na germinação e desenvolvimento inicial de mandacaru (Cereus jamacaru DC.). Agropecuária Técnica, 31(2): 96-101. 
Ferreira MM, Souza JP e Meiado MV. 2014. Morfometria de sementes de duas espécies de Cereus Mill. (Cactaceae). Informativo ABRATES, 24(3): 97.

Figueira JEC, Vasconcellos-Neto J, Garcia MA e Souza ALT. 1994. Saurocory in Melocactus violaceus (Cactaceae). Biotropica, 26(3): 295-301.

Flores J, Jurado E e Arredondo A. 2006. Effect of light on germination of seeds of Cactaceae from the Chihuahuan Desert, Mexico. Seed Science Research, 16(2): 149-155.

Flores J, Jurado E, Chapa-Vargas L, Ceroni-Stuva A, Dávila-Aranda P, Galíndez G, Gurvich D, León-Lobos P, Ordóñez C, Ortega-Baes P, Ramírez-Bullón N, Sandoval A, Seal CE, Ullian T e Pritchard HW. 2011. Seeds photoblastism and its relationship with some plant traits in 136 cacti taxa. Environmental and Experimental Botany, 71(1): 79-88.

Galvão SF, Aleixo LA e Santos DL. 2010. Efeito da qualidade de luz e temperatura na germinação de sementes de Hylocereus undatus Britton \& Rose (Cactaceae). Boletín de la Sociedad Latinoamericana y del Caribe de Cactáceas y otras Suculentas, 10(2): 24-27.

Gomes VGN, Meiado MV, Quirino ZGM e Machado IC. 2016. Seed removal by lizards and effect of gut passage on germination in a columnar cactus of the Caatinga, a tropical dry forest in Brazil. Journal of Arid Environments, 135(1): 85-89.

Guedes RS, Alves EU, Gonçalves EP, Bruno RLA, Braga Júnior JM e Medeiros MS. 2009. Germinação de sementes de Cereus jamacaru DC. em diferentes substratos e temperaturas. Acta Scientiarum. Biological Sciences, 31(2): 159-164.

Guedes RS, Alves EU, Gonçalves EP, Viana JS, Moura MF e Santos SS. 2008. Germinação de sementes de Opuntia inamoena Schum após tratamentos para superar a dormência. Revista de Biologia e Farmácia, 3(1): 66-74.

Kigel J. 1995. Seed germination in arid and semiarid regions. In: Kigel J e Galili G (Eds), Seed development and germination. New York: Marcel Dekker, Inc., p. 645-699.

Langer DF e Mengener RA. 2013. Cultivo in vitro de Cereus hildmannianus K.Schum. Unoesc \& Ciência - ACBS, 4(1): 7-14.

Leal L, Biondi D e Nunes JRS. 2007. Propagação por sementes de Schlumbergera truncata (Haw.) Moran (flor-demaio) em diferentes substratos. Acta Scientiarum. Biological Sciences, 29(3): 277-280.

Lima AR, Cunha MC, Nascimento AMJ e Meiado MV. 2014. Caracterização de um lote de sementes de Melocactus zehntneri (Britton \& Rose) Luetzelb. (Cactaceae) coletado em uma área de Caatinga do Estado de Sergipe. Informativo ABRATES, 24(3): 94.

Lima AT. 2016. Hidratação descontínua altera a germinação de sementes sob estresse de duas populações de cacto que ocorrem em diferentes ecossistemas no Nordeste do Brasil. Monografia. Itabaiana: Universidade Federal de Sergipe. 
Lima AT e Meiado MV. 2014. Efeito da salinidade na germinação de facheiro [Pilosocereus catingicola (Gürke) Byles \& Rowley subsp. salvadorensis (Werderm.) Zappi Cactaceae]. Informativo ABRATES, 24(3): 95.

Lima AT e Meiado MV. 2017. Discontinuous hydration alters seed germination under stress of two populations of cactus that occur in different ecosystems in Northeast Brazil. Seed Science Research, 27(4): 292-302.

Lone AB. 2010. Temperatura e substratos na germinação de sementes de genótipos de pitaya. Dissertação de Mestrado. Londrina: Universidade Estadual de Londrina.

Lone AB, Colombo RC, Favetta V, Takahashi LSA e Faria RT. 2014a. Temperatura na germinação de sementes de genótipos de pitaya. Semina: Ciências Agrárias, 35(4): 2251-2258.

Lone AB, Molo CX, Takahashi LSA e Unemoto LK. 2009. Germinação de sementes de Rhipsalis em diferentes substratos. Scientia Agraria, 10(5): 419-422.

Lone AB, Souza GRB, Oliveira KS, Takahashi LSA e Faria RT. 2010. Temperatura e substrato para a germinação de sementes de flor-de-maio (Schlumbergera truncata (Haw.) Moran). Revista Ceres, 57(3): 367-371.

Lone AB, Takahashi LSA, Faria RT e Unemoto LK. 2007. Germinação de Melocactus babiensis (Cactaceae) em diferentes substratos e temperaturas. Scientia Agraria, 8(1): 365-369.

Lone AB, Unemoto LK, Ferrari EAP, Takahashi LSA e Faria RT. 2014b. The effects of light wavelength and intensity on the germination of pitaya seed genotypes. Australian Journal of Crop Science, 8(11): 1475-1480.

Lopes LT. 2012. Fenologia, biologia reprodutiva, germinação e desenvolvimento inicial de Cipocereus minensis subsp. leiocarpus N.P. Taylor \& Zappi no Planalto de Diamantina - MG. Dissertação de Mestrado. Diamantina: Universidade Federal dos Vales do Jequitinhonha e Murici.

Lucena CM, Carvalho TKN, Ribeiro JES, Quirino ZGM, Casas A e Lucena RFP. 2015. Conhecimento botânico tradicional sobre cactáceas no semiárido do Brasil. Gaia Scientia, 9(2): 77-90.

Lucena CM, Costa GM, Sousa RF, Carvalho TKN, Marreiros NA, Alves CAB, Pereira DD e Lucena RFP. 2012. Conhecimento local sobre cactáceas em comunidades rurais na mesorregião do sertão da Paraíba (Nordeste, Brasil). Revista Biotemas, 25(2): 281-291.

Lucena CM, Lucena RFP, Costa GM, Carvalho TKN, Costa GGS, Alves RRN, Pereira DD, Ribeiro JES, Alves CAB, Quirino ZGM e Nunes EN. 2013. Use and knowledge of Cactaceae in Northeastern Brazil. Journal of Ethnobiology and Ethnomedicine, 9(1): 1-11.

Marchi MNG. 2012. Micropropagação e conservação de Discocactus z̧ehntneri, Pilosocereus gounellei e Stephanocereus luetzelburgii, cactos nativos da Chapada Diamantina, Bahia. Dissertação de Mestrado. Feira de Santana: Universidade Estadual de Feira de Santana.

Marchi MNG, Civatti LM, Viana CM, Assis JGA, Bellintani MC e Santana JRF. 2013. Seed cryopreservation of the native cacti Discocactus zebntneri, Pilosocereus gounellei and Stephanocereus luetzelburgii from Bahia, Brazil. African Journal of Biotechnology, 12(21): 3250-3254. 
Marques VB. 2010. Germinação, fenologia e estimativa de custo de produção de pitaia [Hylocereus undatus (Haw.) Britton \& Rose]. Tese de Doutorado. Lavras: Universidade Federal de Lavras.

Martins LST. 2007. Germinação de sementes de Pilosocereus arrabidae (Lem.) Byl. \& Row (Cactaceae) de Arraial do Cabo, Rio de Janeiro. Dissertação de Mestrado. Rio de Janeiro: Instituto de Pesquisa Jardim Botânico do Rio de Janeiro.

Martins LST, Pereira TS, Carvalho ASR, Barros CF e Andrade CS. 2012. Seed germination of Pilosocereus arrabidae (Cactaceae) from a semiarid region of south-east Brazil. Plant Species Biology, 27(3): 191-200.

Medeiros RLS, Souza VC, Azeredo GA, Pereira EM, Barbosa Neto MA, Medeiros VS e Barbosa AS. 2015. Germinação e vigor de sementes de Pilosocereus catingicola (Gürke) Byles \& Rowley subsp. salvadorensis (Werderm.) Zappi (Cactaceae) da Caatinga paraibana. Gaia Scientia, 9(2): 61-66.

Meiado MV. 2012a. Germinação de cactos do Nordeste do Brasil. Tese de Doutorado. Recife: Universidade Federal de Pernambuco.

Meiado MV. 2012b. Germinação de sementes de cactos do Brasil: fotoblastismo e temperaturas cardeais. Informativo ABRATES, 22(3): 20-23.

Meiado MV. 2012c. Propagação sexual e assexual estruturando populações de Tacinga palmadora (Britton \& Rose) N.P. Taylor \& Stuppy, um cacto endêmico da Caatinga. Revista de Biologia Neotropical, 9(2): 6-13.

Meiado MV. 2013. Evidências de memória hídrica em sementes da Caatinga. In: Stelmann JR, Isaias RMS, Modolo LV, Vale FHA, Salino A (Org), Anais do $64^{\circ}$ Congresso Nacional de Botânica: botânica sempre viva. Belo Horizonte: Sociedade Botânica do Brasil, p. 89-94.

Meiado MV. 2016. Seed germination of Melocactus sergipensis N.P. Taylor \& M.V. Meiado, the newest Brazilian cactus destined for extinction. Plant Species Biology, 31(4): 296-299.

Meiado MV, Albuquerque LSC, Rocha EA e Leal IR. 2008. Efeito da luz e da temperatura na germinação de sementes de Pilosocereus catingicola subsp. salvadorensis (Werderm.) Zappi (Cactaceae). Boletín de la Sociedad Latinoamericana y del Caribe de Cactáceas y otras Suculentas, 5(2): 9-12.

Meiado MV, Albuquerque LSC, Rocha EA, Rojas-Aréchiga M e Leal IR. 2010. Seed germination responses of Cereus jamacaru DC. ssp. jamacaru (Cactaceae) to environmental factors. Plant Species Biology, 25(2): 120-128.

Meiado MV, Aona LYS, Nascimento JPB, Lima AT e Zappi DC. 2015. Cactaceae. In: Prata APN, Farias MCV e Landim MF (Orgs), Flora de Sergipe. Vol. 2. Aracaju: Gráfica Editora Triunfo, p. 115-142.

Meiado MV, Machado MC, Zappi DC, Taylor NP e Siqueira Filho JA. 2012a. Cacti of the São Francisco Watershed: Ecological Attributes, Geographic Distribution and Endemism. In: Siqueira Filho JA (Org), Flora of the Caatingas of the São Francisco River: Natural History and Conservation. Rio de Janeiro: Andrea Jakobsson Estúdio Editorial, p. 264-305.

Meiado MV e Nascimento JPB. 2014. Morfometria e germinação de sementes de Melocactus sergipensis N.P.Taylor 
\& M.V.Meiado (Cactaceae), a mais nova espécie de cacto endêmica e ameaçada de extinção do estado de Sergipe. Informativo ABRATES, 24(3): 95.

Meiado MV, Rojas-Aréchiga M, Siqueira-Filho JA e Leal IR. 2016. Effects of light and temperature on seed germination of cacti of Brazilian ecosystems. Plant Species Biology, 31(2): 87-97.

Meiado MV, Silva FFS, Barbosa DCA e Siqueira Filho JA. 2012b. Diaspore of the Caatinga: A Review. In: Siqueira Filho JA (Org), Flora of the Caatingas of the São Francisco River: Natural History and Conservation. Rio de Janeiro: Andrea Jakobsson Estúdio Editorial, p. 306-365.

Nascimento JPB. 2014. Ecofisiologia da germinação de sementes e estabelecimento de plântulas de Discocactus Pfeiff. (Cactaceae) ameaçados de extinção. Monografia. Petrolina: Universidade Federal do Vale do São Francisco.

Nascimento JPB e Meiado MV. 2017. In situ or ex situ seed conservation: which is the more effective way to maintain seed longevity of an endangered cactus? Plant Species Biology, 32(2): 115-120.

Nascimento JPB, Meiado MV, Nicola PA e Pereira LCM. 2015a. Germinação de sementes de Tacinga inamoena (K. Schum.) N.P. Taylor \& Stuppy (Cactaceae) após endozoocoria por Chelonoidis carbonaria (Spix, 1824) (Reptilia: Testudinidae). Gaia Scientia, 9(2): 9-14.

Nascimento JPB, Vieira DCM e Meiado MV. 2014. Conservação ex situ de sementes de Cactaceae. Informativo ABRATES, 24(3): 87.

Nascimento JPB, Vieira DCM e Meiado MV. 2015b. Ex situ seed conservation of Brazilian cacti. Gaia Scientia, 9(2): 111-116.

Oliveira CAM, Silva AG, Matos VP e Silva MV. 2014. Germinação de sementes de Harrisia adscendens (Gürke) Britton \& Rose, após tratamento pré-germinativo. Informativo ABRATES, 24(3): 85.

Oliveira DM, Lima AT, Rocha EA e Meiado MV. 2017. O aumento da temperatura reduz a tolerância ao estresse hídrico na germinação de sementes de Pereskia grandifolia Haw. subsp. grandifolia (Cactaceae)? Gaia Scientia, 11(4): 26-36.

Ortiz TA, Moritz A, Oliveira MA e Takahashi LSA. 2015. Effects of the hydrogen potential and fungicide treatment on pitaya seed germination. Acta Scientiarum. Agronomy, 37(1): 69-74.

Prisco JT. 1966. Action of light on mandacaru seed germination. Revista Brasileira de Biologia, 26(3): 261262.

Prudente JSN, Ferreira MM, Lima AT e Meiado MV. 2014. Germinação e variação intraespecífica em sementes de facheiro [Pilosocereus catingicola (Gürke) Byles \& Rowley subsp. salvadorensis (Werderm.) Zappi Cactaceae]. Informativo ABRATES, 24(3): 96.

Rebouças ACMN e Santos DL. 2007. Influência do fotoperíodo e qualidade de luz na germinação de sementes de Melocactus conoideus (Cactaceae). Revista Brasileira de Biociências, 5(supl.2): 900-902. 
Rebouças ACMN, Souza AO e Santos DL. 2009. A germinação de sementes de Melocactus conoideus como ferramenta de educação ambiental para crianças moradoras no entorno da Serra do Periperi. Boletín de la Sociedad Latinoamericana y del Caribe de Cactáceas y otras Suculentas, 6(3): 23-27.

Rêgo MM, Araújo ER, Rêgo ER e Castro JP. 2009. In vitro seed germination of mandacaru (Cereus jamacaru DC.). Revista Caatinga, 22(4): 34-38.

Reis MV. 2008. Estudos preliminares do desenvolvimento vegetativo e da germinação de sementes de Cipocereus minensis (Werderm) F. Ritter e Pilosocereus aurisetus (Werderm) Byles \& GD Rowley. Monografia. Diamantina: Universidade Federal dos Vales do Jequitinhonha e Mucuri.

Reis MV, Pêgo RG, Paiva PDO, Artioli-Coelho FA e Paiva R. 2012. Germinação in vitro e desenvolvimento pósseminal de plântulas de Pilosocereus aurisetus (Werderm.) Byles \& G.D. Rowley (Cactaceae). Revista Ceres, 59(6): 739-744.

Resende SV. 2010. Micropropagação e conservação in vitro de Melocactus glaucescens Buining \& Brederoo e Melocactus paucispinus G.Heimen C.R.Paul (Cactaceae), espécies endêmicas da Bahia e ameaçadas de extinção. Tese de Doutorado. Feira de Santana: Universidade Estadual de Feira de Santana.

Resende SV, Lima-Brito A e Santana JRF. 2010. Influência do substrato e do enraizamento na aclimatação de Melocactus glaucescens Buining \& Brederoo propagados in vitro. Revista Ceres, 57(6): 803-809.

Rito KF. 2009. As sementes de mandacaru têm memória hídrica? Monografia. Recife: Universidade Federal de Pernambuco.

Rito KF, Rocha EA, Leal IR e Meiado MV. 2009. As sementes de mandacaru têm memória hídrica? Boletín de la Sociedad Latinoamericana y del Caribe de Cactáceas y otras Suculentas, 6(1): 26-31.

Rojas-Aréchiga M, Orozco-Segovia A, Vázquez-Yanes C. 1997. Effect of light on germination of seven species of cacti from Zapotitlán Valley in Puebla, México. Journal of Arid Environments, 36(4): 571-578.

Rojas-Aréchiga M e Vázquez-Yanes C.2000. Cactus seed germination: a review. Journal of Arid Environments, 44(1): 85-104.

Salles HG. 1987. Expressão morfológica de sementes e plântulas. I. Cephalocereus fluminensis (Miq.) Britton \& Rose. Revista Brasileira de Sementes, 9(1): 73-81.

Sanches LVC, Ferreira MJCL e Bosque G. 2007. Teste de emergência e avaliação de desenvolvimento do cacto Melocactus deinacanthus em diversos tipos de substrato. Revista Científica Eletrônica de Agronomia, 8(12): 1-7.

Santos AP. 2017. Criopreservação e armazenamento de sementes de cactos do Estado de Sergipe. Monografia. Itabaiana: Universidade Federal de Sergipe.

Santos KC e Meiado MV. 2014. Efeito do estresse hídrico na germinação de sementes de Cereus jamacaru DC. subsp. jamacaru (Cactaceae) coletadas em áreas de Caatinga do Estado de Sergipe. Informativo ABRATES, 24(3): 109. 
Secorun AC e Souza LA. 2011. Morphology and anatomy of Rhipsalis cereuscula, Rhipsalisfloccosa subsp. hohenauensis and Lepismium cruciforme (Cactaceae) seedlings. Revista Mexicana de Biodiversidad, 82(1): 131-143.

Silva ACC. 2014. Pitaya: melhoramento e produção de mudas. Tese de Doutorado. Jaboticabal: Universidade Estadual Paulista.

Silva GO. 2009. Germinação de sementes de Pilosocereus gounellei (F.A.C. Weber) Byles \& G.D. Rowley subsp. gounellei (Cactaceae) submetidas a estresse hídrico. Monografia. Recife: Faculdade Frassinetti do Recife.

Silva GO, Nascimento PRF e Meiado MV. 2010. Germinación de semillas de Pilosocereus gounellei (F.A.C. Weber) Byles \& G.D. Rowley subsp. gounellei bajo la influencia del déficit hídrico. Boletín de la Sociedad Latinoamericana y del Caribe de Cactáceas y otras Suculentas, 7(1): 16-20.

Silva NM, Andrade AJP e Souza CR. 2013. O sertanejo e as experiências de inverno no Seridó Potiguar. Desenvolvimento e Meio Ambiente, 27(1): 87-107.

Silveira RA. 2015. Ecologia de Discocactus pseudoinsignis e Discocactus placentiformis simpátricos e endêmicos da Serra do Espinhaço, MG, Brasil. Dissertação de Mestrado. Ouro Preto: Universidade Federal de Ouro Preto. Simão E, Nakamura AT e Takaki M. 2010a. The germination of seeds of Epiphyllum phyllanthus (L.) Haw. (Cactaceae) is controlled by phytochrome and by nonphytochrome related process. Biota Neotropica, 10(1): 115-119.

Simão E, Socolowski F e Takaki M. 2007. The epiphytic Cactaceae Hylocereus setaceus (Salm-Dyck ex DC.) Ralf Bauer seed germination is controlled by light and temperature. Brazilian Archives of Biology and Technology, 50(4): 655-662.

Simão E, Takaki M e Cardoso VJM. 2010b. Germination response of Hylocereus setaceus (Salm-Dyck ex DC.) Ralf Bauer (Cactaceae) seeds to temperature and reduced water potentials. Brazilian Journal of Biology, 70(1): 135-144.

Siqueira Filho JA. 2012. A extinção inexorável do Rio São Francisco. In: Siqueira Filho JA (Org), Flora of the Caatingas of the São Francisco River: Natural History and Conservation. Rio de Janeiro: Andrea Jakobsson Estúdio Editorial, p. 25-65.

Socolowski F, Vieira DCM, Simão E e Takaki M. 2010. Influence of light and temperature on seed germination of Cereus pernambucensis Lemaire (Cactaceae). Biota Neotropica, 10(2): 53-56.

Sousa DMM, Bruno RLA, Andrade AP, Dornela CSM e Brito Primo DM. 2009. Comportamento de sementes de palma (Opuntia ficus-indica L.) submetidas à fermentação e secagem. Revista Caatinga, 22(3): 29-34.

Souza LF, Gasparetto BF, Lopes RR e Barros IBI. 2016. Temperature Requirements for Seed Germination of Pereskia aculeata and Pereskia grandifolia. Journal of Thermal Biology, 57(1): 6-10.

Taylor N, Meiado MV, Bravo Filho E e Zappi D. 2014. A new Melocactus from the Brazilian state of Sergipe. 
Bradleya, 32(1): 99-104.

Taylor N e Zappi D. 2004. Cacti of Eastern Brazil. Kew: The Royal Botanic Garden.

Tomazi EF e Figueiredo RA. 2002. Efeito da ingestão por aves na germinação de sementes de Epiphyllum phyllanthus (Cactaceae) em Jundiaí - SP, Brasil. Argumento, 4(7): 11-15.

Veiga-Barbosa L, González-Benito ME, Assis JGA e Pérez-García F. 2010. Germination and cryopreservation of several cactus species from NE Brazil. Seed Science \& Technology, 38(1): 218-224.

Zamith LR, Cruz DD e Richers BTT. 2013. The effect of temperature on the germination of Melocactus violaceus Pfeiff. (Cactaceae), a threatened species in restinga sandy coastal plain of Brazil. Anais da Academia Brasileira de Ciências, 85(2): 615-622.

Zappi D, Taylor N, Santos MR e Larocca J. 2017. Cactaceae. In: Lista de Espécies da Flora do Brasil. Jardim Botânico do Rio de Janeiro. Disponível em: < http://floradobrasil.jbri.gov.br/reflora/floradobrasil/FB70>. Acesso em: 14 Jun. 2017.

Zimmer K e Büttner P. 1982. Ersatz des licht bedürfnisses bei der keimung von kakteensamen durch gibberellinsäure. Gartenbauwissenschaft, 47(1): 97-101. 